\title{
Calibration of the SBUV version 8.6 ozone data product
}

\author{
M. T. DeLand, S. L. Taylor, L. K.Huang, and B. L. Fisher \\ Science Systems and Applications, Inc. (SSAI), 10210 Greenbelt Road, Suite 600, Lanham, Maryland 20706, USA \\ Correspondence to: M. T. DeLand (matthew.deland@ssaihq.com)
}

Received: 7 June 2012 - Published in Atmos. Meas. Tech. Discuss.: 25 July 2012

Revised: 17 October 2012 - Accepted: 8 November 2012 - Published: 29 November 2012

\begin{abstract}
This paper describes the calibration process for the Solar Backscatter Ultraviolet (SBUV) Version 8.6 (V8.6) ozone data product. Eight SBUV instruments have flown on NASA and NOAA satellites since 1970, and a continuous data record is available since November 1978. The accuracy of ozone trends determined from these data depends on the calibration and long-term characterization of each instrument. V8.6 calibration adjustments are determined at the radiance level, and do not rely on comparison of retrieved ozone products with other instruments. The primary SBUV instrument characterization is based on prelaunch laboratory tests and dedicated on-orbit calibration measurements. We supplement these results with "soft" calibration techniques using carefully chosen subsets of radiance data and information from the retrieval algorithm output to validate each instrument's calibration. The estimated long-term uncertainty in albedo is approximately $\pm 0.8-1.2 \%(1 \sigma)$ for most of the instruments. The overlap between these instruments and the Shuttle SBUV (SSBUV) data allows us to intercalibrate the SBUV instruments to produce a coherent V8.6 data set covering more than $32 \mathrm{yr}$. The estimated long-term uncertainty in albedo is less than $3 \%$ over this period.
\end{abstract}

\section{Introduction}

Satellite instruments using the backscattered ultraviolet (BUV) measurement technique have produced global total column ozone and stratospheric profile ozone data on a daily basis for more than $40 \mathrm{yr}$. The backscattered ultraviolet (BUV) instrument flew on the Nimbus-4 satellite from April 1970 to May 1977, although the data quality and sampling from this instrument is significantly reduced after mid1972 (Stolarski et al., 1997). The Solar Backscatter Ultraviolet (SBUV) instrument on the Nimbus-7 satellite operated from November 1978 to June 1990 (Heath et al., 1975), and seven follow-on SBUV/2 instruments flown on NOAA polarorbiting satellites have collected ozone data from March 1985 to the present (Frederick et al., 1986). Figure 1 shows a timeline of these instruments. Note that four SBUV/2 instruments are currently operational. Data from the NOAA-19 SBUV/2 (launched February 2009) and NPP Ozone Mapping and Profiling Suite (OMPS, launched October 2011) instruments are not discussed here, but are expected to be available in the future. In the remainder of this paper, we will use "SBUV" as a generic term for all of the BUV instruments, with specific reference to individual instruments where appropriate.

Accurate instrument calibration is necessary to produce high quality ozone data for comparison with other measurements (Fioletov et al., 2006), for use in data assimilation systems (Stajner et al., 2004), and for long-term trend analysis (e.g. Stolarski and Frith, 2006; Terao and Logan, 2007). Characterization of each SBUV instrument is intended to use specifically designed measurements in the laboratory and onorbit, including an on-board mercury lamp and solar irradiance measurements, to establish the long-term calibration. Specific aspects of the V8.6 retrieval algorithm output and carefully chosen Earth radiance measurements (restricted by viewing conditions, scene brightness, etc.) can also be used to validate instrument performance. We combine some of these techniques into a "soft" calibration procedure that improves the accuracy and inter-instrument agreement of the V8.6 ozone product.

The focus of this paper is a description of the overall calibration process used to create the SBUV V8.6 ozone data sets. Discussion of the V8.6 algorithm is given in Bhartia et al. (2012), and an overview of the V8.6 results is given in McPeters et al. (2012). A key point of our work is that all calibration adjustments discussed for both spectral and temporal 
Backscattered UV Profile 0zone Measurements

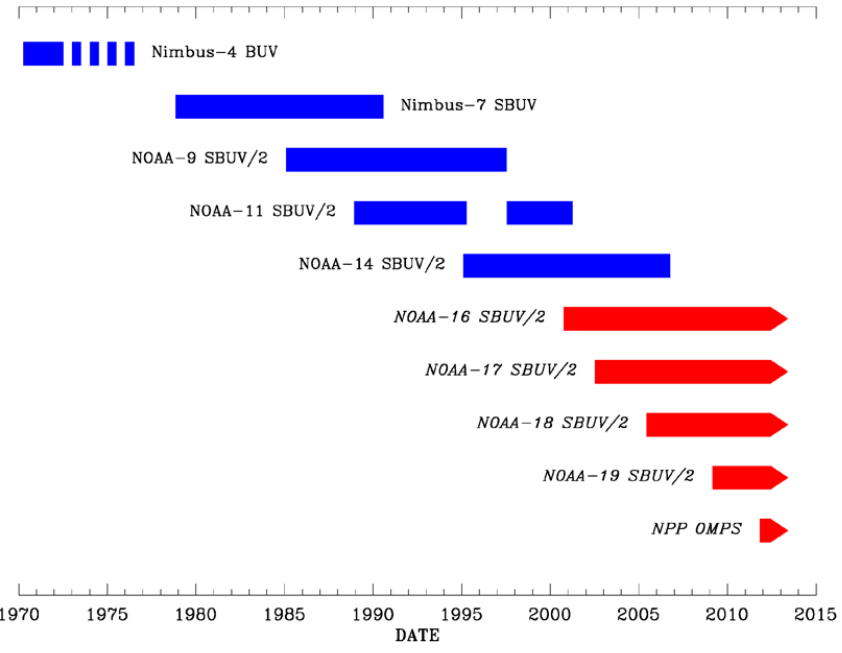

Fig. 1. Timeline of satellite BUV instruments used to measure stratospheric profile and total column ozone for the V8.6 data set. Inactive instruments are shown in blue, and operational instruments are shown in red. The dashed portion of the Nimbus-4 BUV segment indicates the significantly reduced data coverage after July 1972.

variations are derived and applied at the radiance level, rather than being determined from ozone values or other retrieved quantities. The general similarity of design between all of these instruments means that there are many common elements and themes in the calibration process. Nevertheless, there are also many unique circumstances that arise when characterizing the behavior of 8 different instruments over a 40 -yr time period. The Supplement to this paper gives many additional details about the implementation of our calibration process for each instrument, including a comprehensive evaluation of measurement uncertainties.

\section{Characterization of time dependence}

SBUV instruments measure backscattered terrestrial radiance at 12 discrete wavelengths between $252-340 \mathrm{~nm}$ that are selected to characterize the ozone profile from the surface through the upper stratosphere. Regular solar irradiance measurements are also taken (typically weekly) at these wavelengths to provide long-term calibration information (Frederick et al., 1986). Solar activity variations at mid-UV wavelengths can be accurately estimated using the Mg II index and scale factors (DeLand and Cebula, 1993), which are available on a daily basis. The ratio of radiance and irradiance values (defined as directional albedo $=I / F$ ) is the key measured quantity needed for the ozone profile retrieval algorithm. We therefore want to characterize and remove all instrumental effects in the albedo values.
We define the albedo for operational use as the ratio of the observed radiance to the daily solar irradiance, or

$A(\lambda, t)=I(\lambda, t) / F(\lambda, t)$.

The measured radiances incorporate time-dependent variations due to instrument response changes, solar activity, and real geophysical variations. A key design feature of SBUV instruments is that the only mechanical difference in the optical path between radiance and irradiance measurements is the use of a diffuser plate needed to direct solar irradiance into the nadir-viewing instrument aperture. This feature allows monochromator throughput changes and solar activity variations to be cancelled out in the reported albedo values. The raw signal levels for radiance and irradiance measurements at a given wavelength can differ by factors of $10^{3}$ or more, so that accurate characterization of photomultiplier tube (PMT) gain is also required. Thus, once on-orbit changes in diffuser reflectivity and PMT gain are accurately characterized and corrected, the remaining variation in the solar irradiance measurements can be attributed to instrument sensitivity changes, corresponding to monochromator throughput changes. Hilsenrath et al. (1995) discussed this characterization process for the NOAA-11 SBUV/2 instrument. Due to the failure of the NOAA-11 solar diffuser in October 1994, an alternate approach was developed to characterize additional long-term instrument changes after this date, and that approach has been employed for other SBUV/2 instruments as well. We describe both approaches in the following sections.

\subsection{Onboard diffuser reflectivity method}

The reflectivity of the SBUV solar diffuser changes on-orbit as contaminants are darkened due to solar exposure. The Nimbus-4 BUV diffuser was exposed constantly during its mission, and darkened so quickly that the solar measurements could not be used for long-term calibration (Heath and Heany, 1974). The Nimbus-7 SBUV diffuser was exposed only during solar measurements, but did not have an independent system to track diffuser reflectivity changes. These changes were evaluated empirically by Cebula et al. (1988) based on variations in the rate of solar diffuser exposure, but this result was later shown by Herman et al. (1990) to underestimate instrument sensitivity changes. For the SBUV/2 instruments, an on-board calibration system was implemented to track relative changes in diffuser reflectivity using a mercury lamp (Weiss et al., 1991). The instrument alternately views the mercury lamp radiance directly and reflected off the solar diffuser during a calibration sequence, and the ratio of these signals at various emission lines in the $\mathrm{Hg}$ spectrum between $185 \mathrm{~nm}$ and $405 \mathrm{~nm}$ is used to track spectral and temporal changes in diffuser reflectivity. Short-term lamp stability problems prevented the use of these data for NOAA$9 \mathrm{SBUV} / 2$, but the performance of the system was improved for subsequent SBUV/2 instruments. 


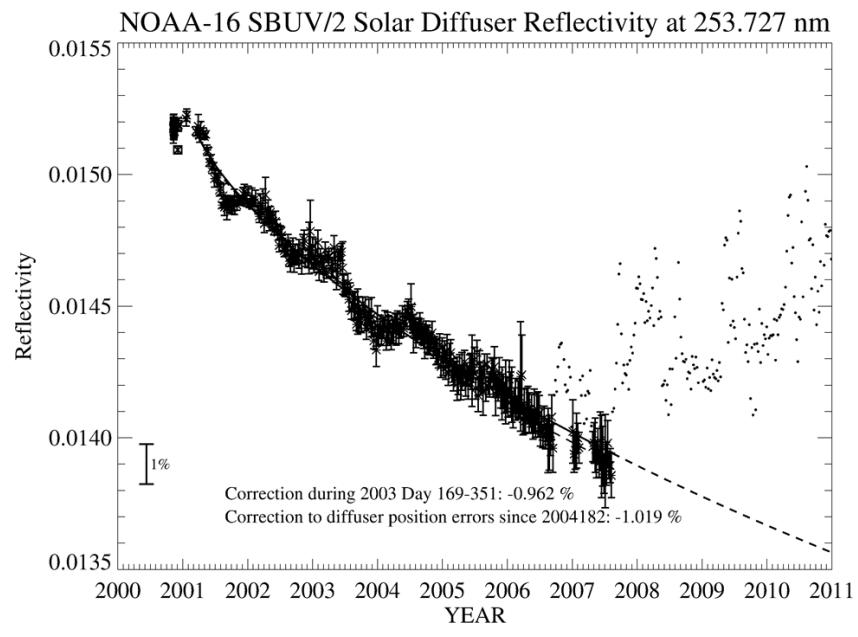

Fig. 2. NOAA-16 SBUV/2 diffuser reflectivity data at $253.7 \mathrm{~nm}$ derived from the onboard mercury lamp calibration system. The solid line is an exponential fit to the data. Note that some measurements in late 2006 and after mid-2007, shown by dots, have been excluded from the analysis due to earthshine contamination at SZA $<120^{\circ}$.

Figure 2 shows an example of the diffuser reflectivity data at $254 \mathrm{~nm}$ for NOAA-16 SBUV/2. Variations in the position of the mercury lamp discharge arc from one calibration sequence to the next can introduce offsets into the lamp output, and thus into the derived diffuser reflectivity. However, regular changes of $0.5-1.0 \%$ caused by the alternating polarity of the Hg lamp power supply (implemented for later SBUV/2 instruments) can be characterized and corrected. The deployment mechanisms for the mercury lamp and the diffuser plate have a \pm 1 step uncertainty in position that can also lead to a shift in the derived diffuser reflectivity for a given sequence. These errors produce wavelength-independent effects in the reflectivity values, and thus can be identified and corrected. The observed diffuser reflectivity changes at the $\mathrm{Hg}$ emission line wavelengths are fit with a smooth function of diffuser exposure time (either linear or exponential) to minimize the impact of short-term fluctuations. Diffuser reflectivity changes are then interpolated to ozone measurement wavelengths for operational use. When the onboard calibration system is fully operational (e.g. NOAA-16, 2001-2008), the estimated longterm calibration accuracy determined by this procedure is approximately $\pm 0.1 \% \mathrm{yr}^{-1}$. Note that NOAA-16 diffuser degradation updates were halted in mid-2008 when the satellite orbit drift led to significant earthshine contamination in the diffuser view measurements. The diffuser degradation correction has been extrapolated during the 2008-2011 period, and we expect to resume useful onboard calibration measurements with this instrument in 2012.

\subsection{Snow/ice radiance method}

An alternative approach to determining long-term instrument sensitivity changes is to identify a stable terrestrial reference target that can be used with radiance measurements. This approach is only practical for SBUV instruments at long wavelengths, due to significant ozone absorption effects at wavelengths shorter than $340 \mathrm{~nm}$. The large field of view (approximately $170 \times 170 \mathrm{~km}$ at the surface) limits the number of potential locations for such an analysis. The Antarctic plateau offers high surface reflectivity from snow and ice-covered surfaces, minimal contamination due to clouds and aerosols, and low terrain height variations to minimize surface bidirectional reflectance distribution function (BRDF) effects. Additional discussion of this technique for satellite UV instruments can be found in Jaross and Krueger (1993), Huang et al. (2003), and Jaross and Warner (2008). While this approach is also applied to measurements of Greenland, the results of the analysis are more uncertain due to the smaller observation region and the increased possibility of aerosol contamination. Thus, the statistical weight of the Greenland data is very low in the SBUV long-term calibration analysis.

Details of the implementation of the snow/ice radiance analysis technique for SBUV instruments are given in Huang et al. (2003), and we summarize key points of this approach here.

1. The analysis uses measurements at the longest SBUV discrete mode wavelength $(340 \mathrm{~nm})$, where the atmospheric ozone absorption effect is small $(<5 \%)$ and easy to correct. We calculate this correction by taking the retrieved total ozone value for each scan and running a forward model radiative transfer calculation to determine the appropriate adjustment. The measured signal is corrected for instrument behavior (electronic offset, non-linearity, thermal sensitivity, PMT gain change) and converted to radiance.

2. The measurement sampling within the defined region of Antarctica (shown in Fig. 3) varies from day to day due to shifting of the satellite orbit track, so data are averaged in approximately weekly (quarter-month) increments for analysis using $1^{\circ}$ solar zenith angle (SZA) bins.

3. A nominal reference year is chosen to define seasonal variations in snow/ice radiance due to SZA variations and surface BRDF effects. Ratios of the radiances at the same SZA and same time of year are computed. This short-term structure is then removed from the snow/ice data for all summer seasons.

4. We estimate sensitivity changes during this reference year using a linear fit to the average radiance change for the preceding and following years.

5. A weighted least squares regression is used to construct a smooth fit to all data points to derive the long-term sensitivity change at $340 \mathrm{~nm}$ (see Fig. 4). Note that the Greenland data are consistent with the linear fit in this example, even though they have relatively little weight 


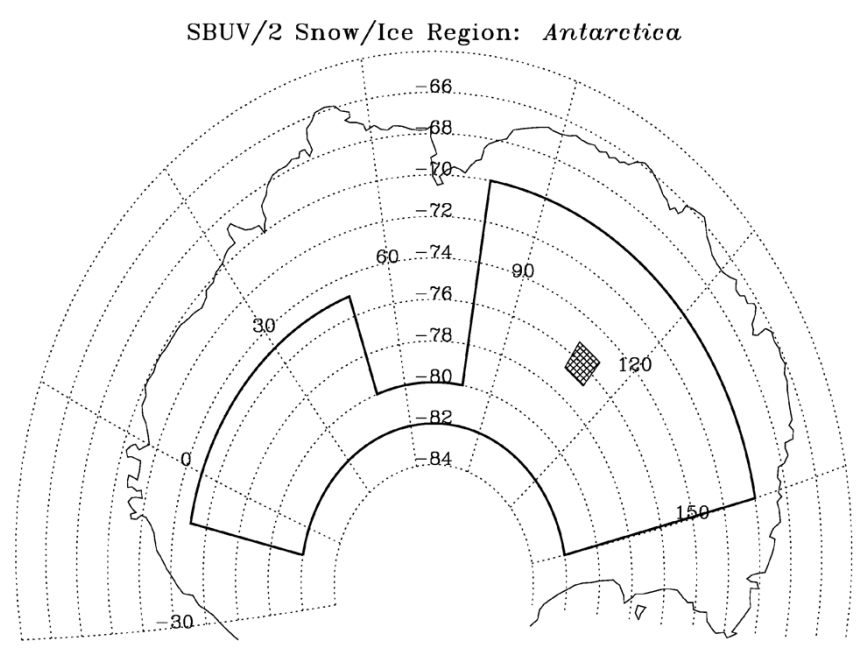

Fig. 3. Region of Antarctica used for SBUV/2 snow/ice albedo calibration measurements (dark outline). The size of a typical SBUV/2 field of view is shown by the filled square.

due to the larger statistical noise. In some cases, multiple linear segments are fit to the data and temporally smoothed, and a final continuous function is then created to represent the sensitivity change.

6. Solar irradiance data (corrected for diffuser reflectivity changes as described in Sect. 2.1) then provides the spectral dependence of instrument sensitivity changes. We adjust the solar-based sensitivity change at $340 \mathrm{~nm}$ to match the snow/ice radiance result, and then apply this adjustment to the observed time series variations at each ozone wavelength. This process allows us to transfer the snow/ice results at $340 \mathrm{~nm}$ to all SBUV wavelengths.

The scatter of averaged and deseasonalized snow/ice samples within a given season is approximately $\pm 0.5 \%$. While this is somewhat less precise than the solar measurements on short time scales, experience has shown that most instrument sensitivity changes have a smooth time dependence over multiyear time periods, so that a simple functional form can be used to characterize the instrument changes represented in snow/ice data. The estimated long-term uncertainty of the fit shown in Fig. 4 is $\sim 0.2 \%$ at the end of 2008, which includes both statistical uncertainties and possible errors in the choice of the functional form. This method does have an advantage compared to the solar-based approach in that mercury lamp behavior, diffuser goniometry effects, and diffuser system performance do not come into play.

The NOAA-11 SBUV/2 diffuser mechanism failed in October 1994, terminating the solar measurements and onboard calibration system measurements. NOAA-11 data collection stopped in April 1995 following the launch of NOAA-14, and did not resume again until July 1997, although the SBUV/2 instrument operated continuously dur-

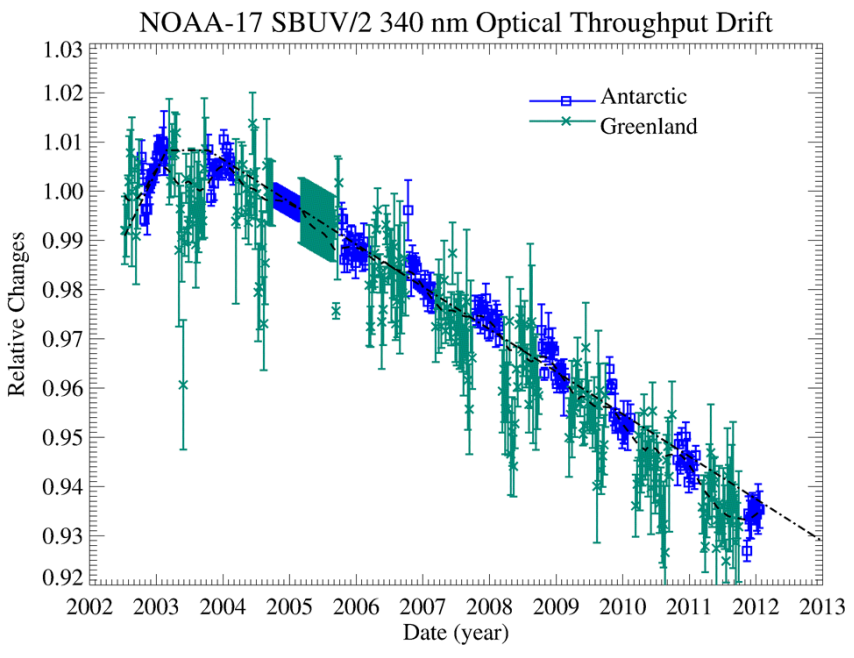

Fig. 4. NOAA-17 SBUV/2 snow/ice albedo data at $340 \mathrm{~nm}$ for characterization of instrument sensitivity time dependence. Squares $=$ Antarctic data. Crosses $=$ Greenland data. All measurements are normalized to October 2002.

ing this time. In order to make an extension of the long-term calibration across this gap, we assume that the throughput change has an exponential dependence on accumulated optical surface contaminants (Stewart et al., 1989). We then used the spectral dependence determined from the on-board calibration system in 1993-1994, and represent the sensitivity change as

$S(\lambda, t)=S\left(\lambda_{0}, t\right) \wedge\left[\operatorname{Ln}\left(S_{1}\left(\lambda, t_{1}\right)\right) / \operatorname{Ln}\left(S_{1}\left(\lambda_{0}, t_{1}\right)\right)\right]\left(t>t_{1}\right)$

$\lambda_{0}=340 \mathrm{~nm} ; t_{1}=$ December $1994 ; S\left(\lambda_{0}, t\right)=$ Sensitivity change at $\lambda_{0}$ from snow/ice analysis; $S_{1}\left(\lambda, t_{1}\right)=$ Sensitivity change at $\lambda$ normalized to snow/ice analysis results at $t_{1}$.

This approach reduces the NOAA-11 calibration uncertainty from the results previously presented in Huang et al. (2003).

\subsection{Other time-dependent corrections}

\subsubsection{Inter-range gain ratio}

The SBUV instrument uses three electronic gain ranges to measure radiance signals over six orders of magnitude, as shown in Fig. 5. Range 1 and Range 2 signals are read from the PMT anode, while Range 3 signals can be read from either the anode or cathode for the most recent SBUV/2 instruments (see Supplement for details). These gain ranges are read out simultaneously and have some overlap in dynamic range, so that selected measurements can give valid data in two gain ranges simultaneously. DeLand et al. (2001) discusses this capability in more detail. Since the PMT cathode is not expected to change response over time, we can therefore use the ratio between Range 2 PMT anode and Range 3 PMT cathode signals $\left(\operatorname{IRR}_{23}\right)$ as a means to track 


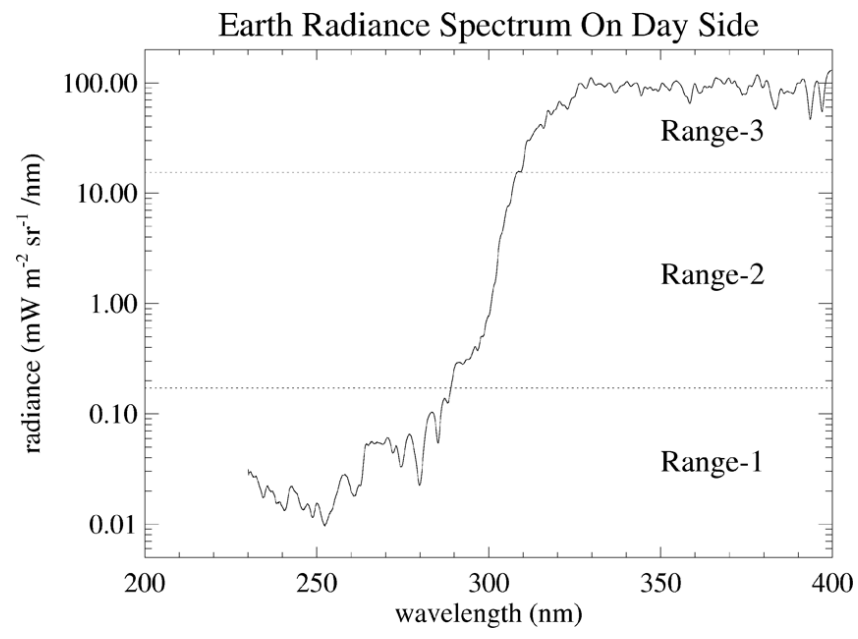

Fig. 5. Backscattered terrestrial radiance spectrum at ultraviolet wavelengths observed by an SBUV instrument. The approximate transition levels between electronic gain ranges are also shown.

time-dependent changes in PMT gain. While the overall PMT gain change can vary from $7 \%$ to $25 \%$ over the lifetime of each instrument, we can characterize this change to $\sim 0.2 \%$ accuracy.

\subsubsection{Goniometry}

The reflectivity of the solar diffuser has a significant angular dependence at the high incidence angles $\left(\theta=55^{\circ}-80^{\circ}\right)$ used for SBUV irradiance measurements. A goniometric correction is derived from prelaunch calibration data and applied during the processing of these measurements. The spectral dependence of this correction is weak over the ozone measurement wavelength range (less than $1 \%$ ), and is characterized using special observations during the on-orbit validation period. However, as the on-orbit azimuth angle changes with time due to satellite orbit drift, errors in the prelaunch goniometric correction frequently appear that can introduce time-dependent errors into the solar irradiance values. These errors have a distinct seasonal variation, and can be characterized using on-orbit data, as demonstrated by Hilsenrath et al. (1995). We require this correction to reduce the goniometry errors to $<0.5 \%$ to avoid any impact on the derived time-dependent instrument characterization. The impact on the sensitivity fit is $<0.3 \%$ when this goal is achieved.

\subsection{Long-term calibration performance and validation}

The long-term SBUV instrument characterization results obtained using the snow/ice radiance technique are generally comparable to results derived from the on-board calibration system at $340 \mathrm{~nm}$. The agreement between these methods is excellent (within $\sim 0.5 \%$ ) for NOAA-14 and NOAA-16, so the calibration system results are used for V8.6 processing. Larger differences of 2-3\% in $340 \mathrm{~nm}$ sensitivity change be- tween onboard calibration and snow/ice radiance results during the first year of operations were observed for NOAA-11, NOAA-17, and NOAA-18. Comparison of equatorial radiance measurements at $252-288 \mathrm{~nm}$ using the $\mathrm{C}-\sigma$ technique described by McPeters (1980), which assumes an exponentially varying ozone profile above $3 \mathrm{hPa}$, gives similar sensitivity changes to the ice radiance results during early operations for NOAA-17 and NOAA-18. This consistency between short wavelength and long wavelength results implies wavelength-independent calibration errors, as discussed in Sect. 2.1. These results were validated by comparison with the concurrent NOAA-16 observations. Thus, the snow/ice results have been adopted for V8.6 processing of NOAA-17 and NOAA-18 data.

The Supplement to this article presents additional discussion of SBUV instrument time-dependent characterization and estimated uncertainty values. The estimated relative albedo uncertainty at the end of the data record for NOAA11 through NOAA-18 is approximately $\pm 0.8-1.2 \%(1 \sigma)$ at all wavelengths. Earlier SBUV instruments did not have on-board calibration data, either because no system existed (Nimbus-4 BUV and Nimbus-7 SBUV) or because of performance problems (NOAA-9 SBUV/2). The long-term characterization procedures used for these instruments are discussed in more detail in the Supplement. These instruments (particularly NOAA-9) have larger estimated time-dependent uncertainty values.

\section{Absolute calibration}

The SBUV instruments undergo radiometric tests before launch in both radiance and irradiance configuration. The radiance measurements use an external flat plate diffuser to illuminate the instrument aperture, whereas the irradiance measurements use the solar diffuser for this purpose. For SBUV/2 instruments, all laboratory sources are directly traceable to NIST reference standards. The estimated accuracy of these irradiance sources is $\sim 2-3 \%(2 \sigma)$ at $340 \mathrm{~nm}$ (Walker et al., 1987). However, since the radiance and irradiance calibrations typically use the same lamps, the lamp irradiance cancels out in the determination of the prelaunch albedo calibration. The most significant element of the albedo calibration then becomes the BRDF of the flat plate diffuser used for the laboratory radiance calibration. The accuracy and stability of the BRDF calibration has improved during the SBUV program (Janz et al., 1995; Butler et al., 2003), and internally illuminated integrating spheres were used for the most recent laboratory calibrations (Heath et al., 1993). The BRDF spectral precision is approximately 0.5 $1.0 \%$ for the most recent SBUV/2 instruments. 


\subsection{Evaluation of prelaunch calibration}

The first step in on-orbit evaluation of the prelaunch albedo calibration is to verify the reflectance of the solar diffuser prior to use. We found changes less than $\pm 1 \%$ relative to prelaunch laboratory data, which is within the uncertainty of a single on-orbit measurement, so we assumed no change in reflectance. The initial "Day 1" solar irradiance measurement, processed with the laboratory calibration, was then compared with previous solar reference measurements such as SSBUV (Cebula et al., 1996). These comparisons showed spectrally dependent differences of 5-10\% for early SBUV instruments (Cebula et al., 1991), and up to $12 \%$ for some recent instruments, suggesting changes in monochromator sensitivity following the final laboratory calibration. However, since the ozone retrieval algorithm uses albedo values, we can examine Antarctic snow/ice albedo data at $340 \mathrm{~nm}$ to determine if the prelaunch albedo calibration is still valid. The NOAA-17 and NOAA-14 measurements were in good agreement with previous results from Nimbus-7 TOMS and EarthProbe TOMS, so we made no change to the laboratory calibration for those instruments. NOAA-16 and NOAA18 Antarctic albedo values were $5.7 \%$ and $7.6 \%$ lower than concurrent data from NOAA-17, respectively. In addition, NOAA-16 and NOAA-18 $340 \mathrm{~nm}$ measurements over open ocean selected to give minimum surface reflectivity (Herman et al., 1991) produced negative values when processed with the prelaunch calibration. As noted in Sect. 2.4, similar offsets were also observed in short wavelength albedo comparisons for these instruments. We therefore applied a wavelength-independent adjustment to the NOAA-16 and NOAA-18 calibration based on the comparisons at $340 \mathrm{~nm}$ with NOAA-17. This implies that the overall spectral dependence of the prelaunch albedo calibration has not changed. We assign an uncertainty of $0.5 \%$ to the albedo calibration for this adjustment.

Although we can validate the large-scale consistency of the prelaunch albedo calibration, the V8.6 algorithm response to an albedo error in a single channel has a complex dependence on the spectral location of that channel, as well as measurement conditions such as solar zenith angle and total ozone. Such errors can lead to incorrect trends in layer ozone time series because of the shift in contribution function location induced by the drifting orbits of many SBUV/2 instruments. Thus, a careful examination of possible calibration errors on a channel-by-channel basis using on-orbit data is required to create the best SBUV ozone product. The following section describes this procedure in more detail.

\subsection{Validation and soft calibration}

An important part of the V8.6 calibration process is the validation of the Level 2 data products. We employ a number of specific techniques, which use both carefully selected measurements and information produced by the V8.6 algorithm, to accomplish this validation. By applying these techniques in a specific sequence, we have developed a "soft" calibration procedure (as distinguished from the "hard" calibration based on laboratory measurements and dedicated in-flight systems) that allows us to evaluate spectrally dependent calibration adjustments at a level of accuracy greater than what laboratory standards can provide.

The first step in the soft calibration procedure is the evaluation of $340 \mathrm{~nm}$ albedo data over snow and ice-covered regions of Antarctica, as described in Sects. 2.2 and 3.1. The NOAA-17 SBUV/2 prelaunch calibration at $340 \mathrm{~nm}$, which is directly tied to the laboratory standards, was established as the benchmark for the V8.6 data product. The NOAA-16 and NOAA-18 calibration values at $340 \mathrm{~nm}$ were adjusted to obtain agreement with NOAA-17, and this adjustment was applied to all wavelengths for each instrument. NOAA-14 and NOAA-11 calibration adjustments at $340 \mathrm{~nm}$ were determined from coincidence analysis with SSBUV underflights, as described further in Sect. 3.3. NOAA-9 and Nimbus-7 calibration adjustments at $340 \mathrm{~nm}$ were determined from coincidence analysis with NOAA- 11 .

The next step in the soft calibration procedure is the determination of individual channel adjustments for profile ozone retrievals. The V8.6 retrieval algorithm determines an a priori ozone profile for each measurement using climatology information from McPeters and Labow (2012) and an initial estimate of total column ozone based on B-pair $(317.5 \mathrm{~nm}, 331.2 \mathrm{~nm})$ data, and then calculates estimated radiances at each wavelength for the observation conditions of that measurement using a forward model. The differences between measured and calculated radiances at each wavelength are called radiance residuals. The profile retrieval minimizes the radiance residuals in several iterations. We use the term "final residuals" to denote the differences between the measurements and the theoretical radiance calculated from the converged retrieved ozone profile. These residuals are typically expressed in terms of $\mathrm{N}$-value units, where $N=-100 \log _{10}(I / F)$, and the albedo $I / F$ is defined in Eq. (1). The set of consecutive channels to be used for each individual retrieval, excluding the surface reflectivity channel at $331.2 \mathrm{~nm}$, is determined automatically based on the slant optical path, which depends on solar zenith angle and total ozone. The shortest channel used in V8.6 retrievals is channel $2(273.5 \mathrm{~nm})$. The longest channel used in the retrieval can vary from channel $7(301.9 \mathrm{~nm})$ to channel 10 $(317.5 \mathrm{~nm})$, depending on measurement conditions.

If the final residuals for the set of channels used in an ozone profile retrieval are greater than approximately \pm 0.1 $\mathrm{N}$-value, then the error is large enough to cause a conflict between the individual channel radiances. This conflict may represent an error in the prelaunch calibration at one or more wavelengths, an error in the radiative transfer portion of the retrieval algorithm, measurement noise at one or more wavelengths, or some combination of these effects. Note that a channel with a non-zero final residual does not necessarily 
have a calibration error. The goal of the soft calibration procedure is to minimize these conflicts.

The following discussion focuses on the implementation of this technique for the NOAA-17 SBUV/2 instrument. We typically create weekly average, 5 degree zonal mean final residual products for this validation step, following the assumption that statistical averaging will reduce the contribution from measurement noise. Observations at equatorial latitudes (corresponding to low total ozone values) and low solar zenith angles produce the maximum overlap between channel weighting functions. We focus on low reflectivity scenes to reduce contamination from clouds. Our validation standard for this analysis is that the average final residual for each channel be smaller than $\pm 0.1 \mathrm{~N}$-value. We found that multiple channels exceeded this threshold when processed with the prelaunch radiance calibration. Figure 6 shows an example of the identification and correction of radiance errors using this analysis. We select the channel with the largest final residual discrepancy, and then reprocess the data set with this channel excluded from the retrieval, using only measurements that satisfy the maximum weighting function overlap conditions (Fig. 6a). This special processing yields a larger average final residual for the excluded channel, which corresponds to an adjustment relative to the initial calibration. This analysis is repeated for any additional channels with final residuals that exceed the threshold, as shown in Fig. 6 b. When all individual channel adjustments determined through this iterative analysis are used together, the final residuals calculated from normal ozone processing will be below the $\pm 0.1 \mathrm{~N}$-value threshold (Fig. 6c). We note that this set of soft calibration adjustments for a specific instrument such as NOAA-17 is not necessarily unique, and may not exactly correspond to errors of the same sign and magnitude in the prelaunch calibration, since final residuals can also incorporate radiative transfer calculation errors.

The preceding discussion can be used to determine calibration adjustments for channels $2-7$. As noted previously, the V8.6 algorithm will automatically extend the wavelength range for a profile retrieval to use channel $8(305.8 \mathrm{~nm}), 9$ $(312.5 \mathrm{~nm})$, or $10(317.5 \mathrm{~nm})$ based on measurement conditions. To determine calibration adjustments to minimize the final residuals for these channels, we first identify a narrow range in latitude (approximately five degrees) where channel 7 rapidly transitions from consistently being the longest channel in the profile retrieval to $50 \%$ or less frequency as the longest channel, based on annual average ozone measurements. The average final residual for channel 8 within this latitude range is adopted as the calibration adjustment for that channel. Subsequent intermediate data processing then allows us to repeat this analysis for channel 9 (using channel 8 as the longest channel) and channel 10 (using channel 9 as the longest channel). The procedure described in this section was used to derive radiance calibration adjustments between $-0.6 \%$ and $+1.4 \%$ at three channels $(283.1 \mathrm{~nm}, 292.3 \mathrm{~nm}$, $317.5 \mathrm{~nm}$ ) for NOAA-17 SBUV/2.
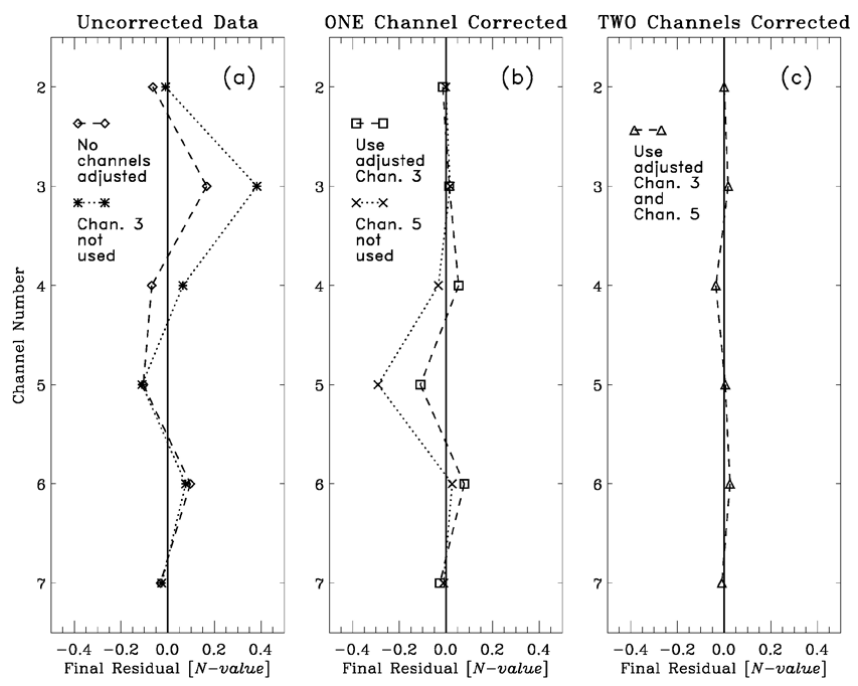

Fig. 6. Determination of individual channel calibration adjustments using final residuals. All results are based on NOAA-17 data averaged over the Pacific Ocean and equatorial latitudes, using only low reflectivity measurements $(R<10 \%)$. (a) Diamonds show residuals with no adjustments. Asterisks show residuals when chan. 3 $(283.1 \mathrm{~nm})$ is excluded from retrieval processing. (b) Squares show residuals when chan. 3 calibration is adjusted based on the error shown in the "excluded" case from (a). Crosses show the residuals when chan. $5(292.3 \mathrm{~nm})$ is excluded from this processing. (c) Triangles show residuals when the calibration for both chan. 3 and chan. 5 is adjusted based on the errors shown in the "excluded" cases.

When we applied the individual channel adjustment approach to other SBUV instruments, we found that we could achieve comparable results for some instruments (e.g. NOAA-18), but that other instruments had significantly larger uncertainties. Since a key goal of the V8.6 ozone product is to create individual data sets that can be merged smoothly for long-term trend studies, we felt that it was more effective to use a minimum number of reference instruments and derive further adjustments based on overlap periods. This approach also reduces the overall data set uncertainty, because the calibration uncertainty associated with the intercalibration process can be less than the absolute uncertainty for some early SBUV instruments.

\subsection{SSBUV coincidence analysis}

When the SBUV/2 series of instruments was implemented as an operational NOAA program in the 1980 s, it was realized that intercalibrating these instruments would be essential to constructing an accurate long-term ozone record. One approach to intercalibration is to have regular underflights with a reference instrument whose calibration can be carefully tracked before, during, and after each flight. The engineering model of the SBUV/2 instrument was converted to fly on the Space Shuttle and renamed Shuttle SBUV (SSBUV) for this purpose (Frederick et al., 1990). Hilsenrath et 
al. (1995) describe the use of SSBUV coincidence measurements for calibration of NOAA-11 V6 ozone data in detail. For the V8.6 ozone data set, the NOAA-11 calibration was revised based on comparisons with SSBUV-2 and SSBUV-6 data taken in October 1990 and March 1994, respectively. We then determined calibration adjustments for NOAA-9 and Nimbus-7 data based on coincidence comparisons to these adjusted NOAA-11 data. In practice, we found that the number of useful samples for this analysis (after satisfying geographical and temporal coincidence requirements) is significantly reduced by the need for steady scene reflectivity in the cloud cover radiometer (CCR) samples taken at $378.6 \mathrm{~nm}$ every two seconds during each scan. We required $\Delta R_{\mathrm{CCR}}<1 \%$ during the scan for our analysis. This limitation is reflected in the estimated uncertainty for these coincidence adjustments, which ranges from approximately $0.7 \%$ at short wavelengths to $2.0 \%$ at long wavelengths, as shown in Hilsenrath et al. (1995).

\subsection{Intercalibration of instruments}

The temporal overlap between SBUV instruments provides an opportunity to intercalibrate these data sets in a continuous chain covering a significant portion of the V8.6 data record. One option is to compare zonal average values in a region with low natural variability, such as the tropics. However, the drift in satellite Equator-crossing time for many instruments (shown in Fig. 7) complicates such an analysis. Note that the onset of rapid orbit drift is delayed for NOAA-16 and subsequent SBUV/2 instruments as a result of a change in the satellite launch procedure (Price, 1991). Overlapping data sets from SBUV instruments typically have a significant difference in local time for a specified latitude band, which can introduce diurnal variations of 2-8\% into the retrieved upper stratospheric ozone profile (Boyd et al., 2007; Haefele et al., 2008). The latitude and seasonal dependence of these variations is not sufficiently understood to adequately simulate and remove this contribution to the observed radiances in simple zonal mean comparisons.

An alternative approach (for polar-orbiting satellites) is provided by a geographically restricted method such as the Simultaneous Nadir Overpass (SNO) technique (Cao et al., 2004). This technique takes advantage of the fact that polarorbiting satellites in Sun-synchronous orbits will have geographic coincidences at high latitudes $\left(70^{\circ}-80^{\circ}\right)$ in both hemispheres. The frequency of temporal coincidences depends on the orbital altitude of the two satellites being considered. For our work with SBUV/2 instruments, which make only daytime measurements, the statistical sampling was improved when we selected situations where one instrument is in an ascending node daytime orbit and the other instrument is in a descending node orbit. This choice results in two sets of measurements, occurring during local summer in each hemisphere, where our "no local time difference" (NLTD) requirements are satisfied. We selected measurements from

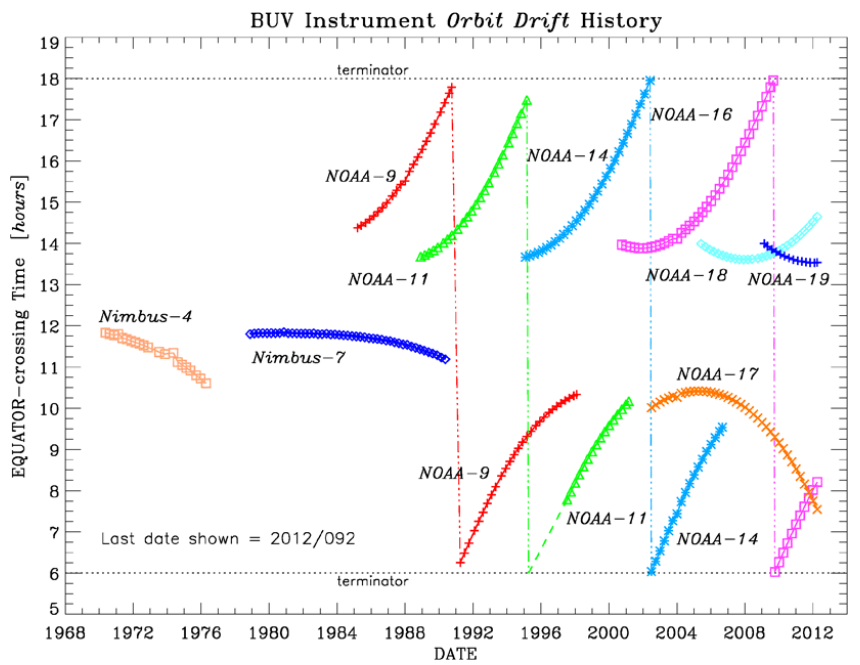

Fig. 7. Time series of Equator-crossing (EC) time for daytime observations for all BUV instruments through April 2012. EC times between 12:00-18:00 h (afternoon orbit) correspond to ascending node daylight measurements. EC times between 06:00-12:00 h (morning orbit) correspond to descending node daylight measurements. Dot-dash lines show when an instrument transitioned from afternoon orbit to morning orbit.

each satellite taken within $30 \mathrm{~min}$ and $1.5^{\circ}$ in latitude for use in this analysis, and also required that the integrated total ozone column from the profiles differ by less than 1.5 Dobson units (DU). We found that Southern Hemisphere NLTD data typically occurred at higher solar zenith angles than Northern Hemisphere NLTD data, and that Southern Hemisphere results were significantly noisier when examining the set of measurements for an entire season. We therefore restricted our intercalibration analysis to Northern Hemisphere NLTD data for the creation of this V8.6 data set.

The NOAA-17 data set was selected as a reference, for reasons discussed previously in Sect. 3.2. Comparisons of NOAA-18 NLTD data to NOAA-17 data for six Northern Hemisphere summer seasons between 2005-2010 are shown in Fig. 8. Since there is no clear temporal signature in these results, we averaged all years together to create relative adjustments for NOAA-18 data at channels 210 . These averaged values are relatively small, and are less than $\pm 0.2 \mathrm{~N}$-value for all channels. The NLTD adjustments are then applied in conjunction with the snow/ice adjustment (wavelength-independent) to create the revised NOAA18 calibration for V8.6 processing. A similar analysis was applied to determine NOAA-16 intercalibration adjustments relative to NOAA-18, using overlap data from 2002-2010. NOAA-14 intercalibration adjustments were then derived from NLTD comparisons with adjusted NOAA-16 data during 2001-2005. The intercalibration adjustments for NOAA14, NOAA-16, and NOAA-18 are shown in Fig. 9.

Extending the NLTD approach to NOAA-11 proved to be difficult, because NOAA-11 and NOAA-16 have only 


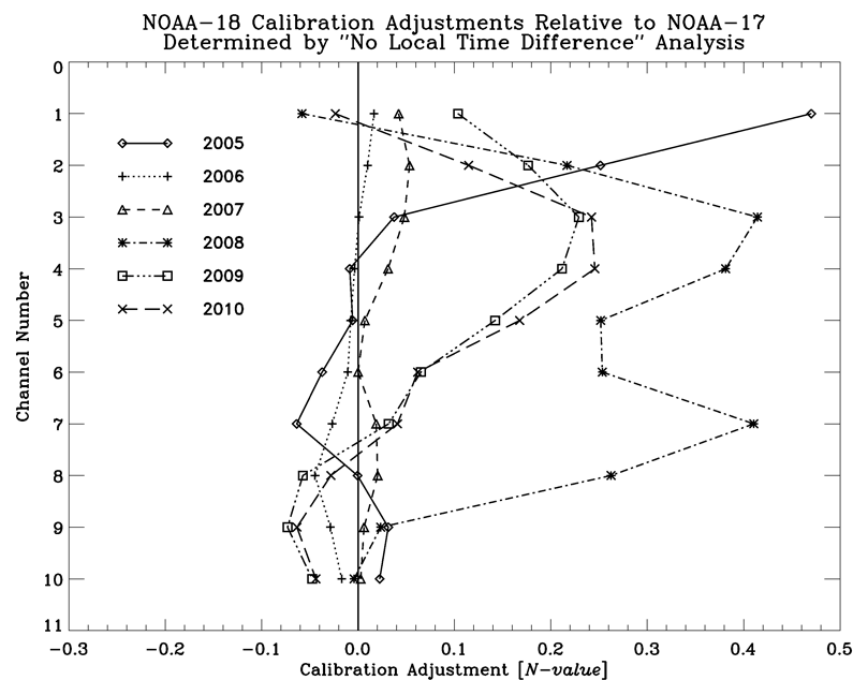

Fig. 8. NOAA-18 calibration adjustments as a function of wavelength relative to NOAA-17 for the years 2005-2010, as determined using the "no local time difference" (NLTD) method. Coincident profiles used for this analysis were taken within $1.5^{\circ}$ latitude and 30 min of each other, and have no more than 1.5 DU difference in total ozone value.

one Southern Hemisphere overlap period in 2000-2001, and the NOAA-11 overlap period with NOAA-14 in July 1997 had poor data quality. We therefore used NOAA-11 calibration adjustments based on coincident measurements with SSBUV, as described in Sect. 3.3. NOAA-9 and Nimbus-7 intercalibration adjustments were then determined from comparisons with adjusted NOAA-11 data. The intercalibration adjustments for Nimbus-7 and NOAA-9 are shown in Fig. 10. Note that the Nimbus- 7 curve also includes an adjustment to all wavelengths based on $340 \mathrm{~nm}$ snow/ice comparisons. Additional coincidence comparison studies including the early SBUV instruments have been performed, but the results were not used in the determination of calibration changes for the V8.6 data product. Figure 11 illustrates the calibration chain for the different SBUV instruments in schematic form.

The improvement in ozone profile data quality produced by the calibration changes discussed in this section is noticeable when examining data from the most recent SBUV instruments on NOAA-16, NOAA-17, and NOAA-18. Figure 12 shows zonal average profile ozone comparisons with Aura MLS Version 3.3 ozone data, using both the previous SBUV V8 data product (top panel) and the current V8.6 data product (bottom panel). The significant decrease in oscillating vertical structure, or "ringing", for the V8.6 comparisons comes from the channel adjustment procedure used for NOAA-17. The improved agreement between these three SBUV instruments in the V8.6 product comes from the use of the "no local time difference" intercalibration technique.

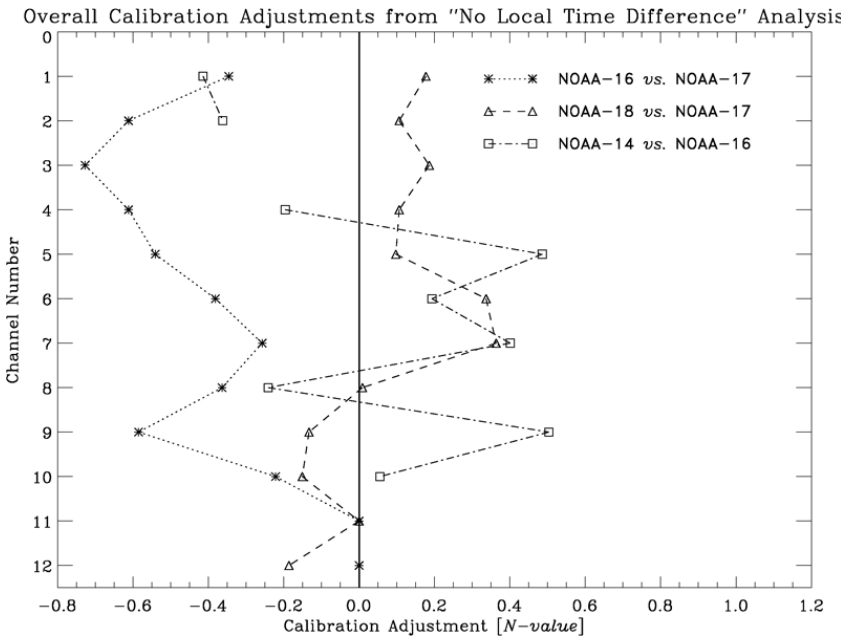

Fig. 9. Calculated intercalibration adjustments for NOAA-16 and NOAA-18 relative to NOAA-17, and for NOAA-14 relative to NOAA-16, based on "no local time difference" coincidence analysis.

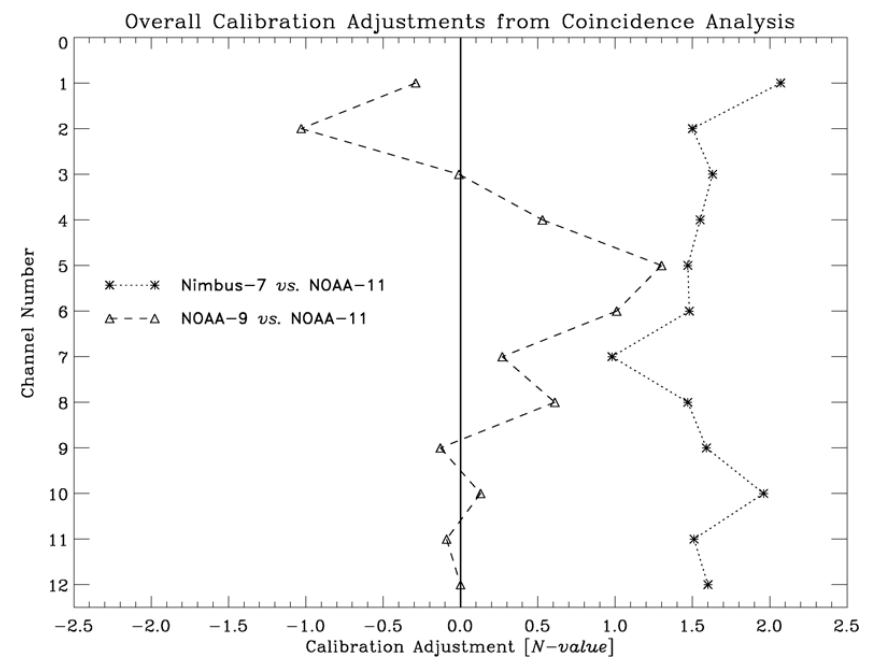

Fig. 10. Calculated intercalibration adjustments for NOAA-9 and Nimbus-7 relative to NOAA-11, based on coincidence comparisons at equatorial latitudes. Note that the Nimbus-7 adjustment also includes a wavelength-independent adjustment of $-4 \%$ based on Antarctic snow/ice radiance data.

\section{Instrument-specific issues}

Additional artifacts are observed in some SBUV measurements that only affect one or more instruments, but nevertheless require significant corrections to the observed radiances. This section describes the most significant issues and how corrections have been determined. 


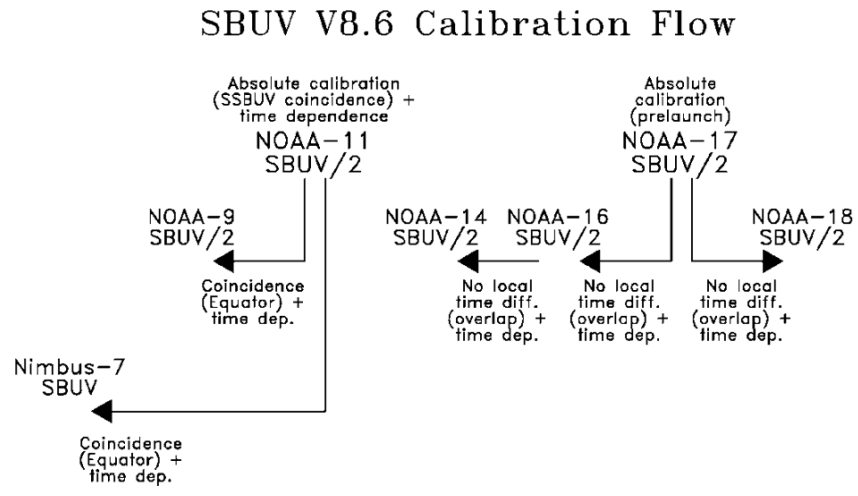

Fig. 11. Schematic diagram of calibration flow for SBUV instruments represented in V8.6 ozone product (except Nimbus-4 BUV).

\subsection{Hysteresis}

The input radiance signal for an SBUV instrument photomultiplier tube (PMT) increases by four orders of magnitude in approximately $5 \mathrm{~min}$ when the instrument first comes out of darkness on each orbit. If the PMT gain does not respond quickly to this change, anode signals will be lower than expected for such conditions (observed at high Southern Hemisphere latitudes for an instrument in an afternoon Equator-crossing orbit) until the PMT response stabilizes. The term "hysteresis" is used here to describe this effect because the PMT current history affects the observed response. PMT cathode signals are not affected by the hysteresis problem. While this effect can be approximately parameterized as a function of solar zenith angle, surface conditions with higher or lower radiance values along the orbit track (e.g. bright clouds or ice, dark ocean) will also influence the PMT current history for that orbit, and thus the magnitude of correction needed.

A previous analysis of this problem was published for NOAA-9 SBUV/2 (DeLand et al., 2001). Significant effects were only observed shortly after crossing into daylight for PMT anode signals (gain ranges 1 and 2). Since PMT cathode data in gain range 3 were not affected, we used interrange ratio data (described in Sect. 2.3.1) to characterize the error. The hysteresis effect was characterized as a quadratic function of SZA for each day, with a maximum radiance correction of $\sim 2 \%$ at $\mathrm{SZA}=85^{\circ}$, decreasing to no correction at $\mathrm{SZA}=65^{\circ}$. For NOAA- 11 and subsequent SBUV/2 instruments, on-orbit analysis shows $<0.5 \%$ maximum hysteresis effect for PMT anode measurements, so no correction has been applied to these data.

The Nimbus-7 SBUV instrument has a significantly larger hysteresis error (maximum effect $=8-9 \%$ ), which affects all measurements because all data from this instrument were collected from the PMT anode. This error can be characterized using concurrent samples collected by a photodiode, which receives approximately $10 \%$ of the incoming radiation through the use of a splitting mirror. This photodiode (oper-
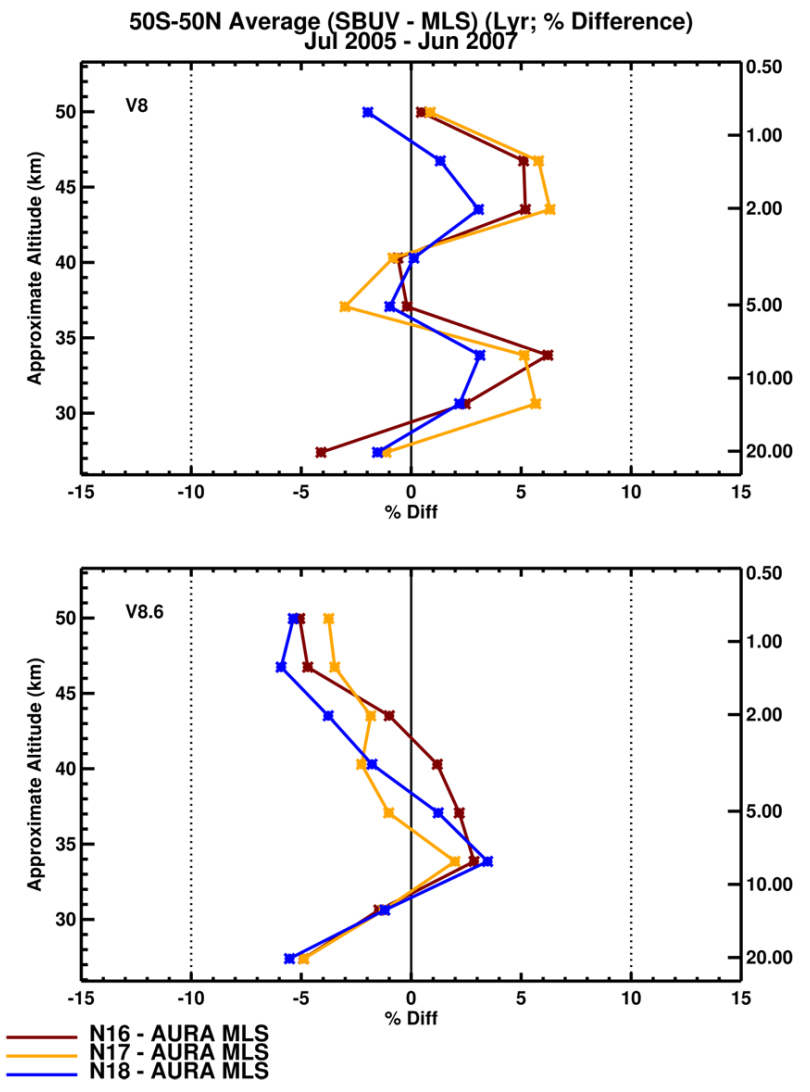

Fig. 12. Profile ozone differences [in percent] between Aura MLS V3.3 data and SBUV/2 instruments on NOAA-16, NOAA-17, and NOAA-18, using zonal averages between $50^{\circ} \mathrm{N}-50^{\circ} \mathrm{S}$ for the period July 2005-June 2007. Comparisons to the SBUV V8 data product are shown in the top panel, and comparisons to the SBUV V8.6 product discussed in this paper are shown in the bottom panel.

ating at $343 \mathrm{~nm}$ ) is not affected by the PMT problem, and thus can be used as a reference to quantify the hysteresis error. We used the accumulated PMT counts during each orbit as a proxy for PMT anode current to develop an effective parameterization of the hysteresis effect. The resulting correction can reach zero at relatively high latitudes during Southern Hemisphere summer when early orbit measurements are taken over the bright Antarctic continent (Fig. 13a), or extend to the Equator in Southern Hemisphere winter when early orbit measurements are taken over the much darker ocean (Fig. 13b). The magnitude of the Nimbus-7 SBUV hysteresis correction also varied over time, with values in 1990 only half as large as those observed in 1979-1980. Figure 14 shows the calculated time dependence of the hysteresis correction at a signal level of 10000 accumulated counts, which typically corresponded to $\mathrm{SZA} \approx 83^{\circ}-85^{\circ}$. The estimated accuracy of this correction, which is applied equally to all wavelengths for an affected scan, is approximately $0.5 \%$. 


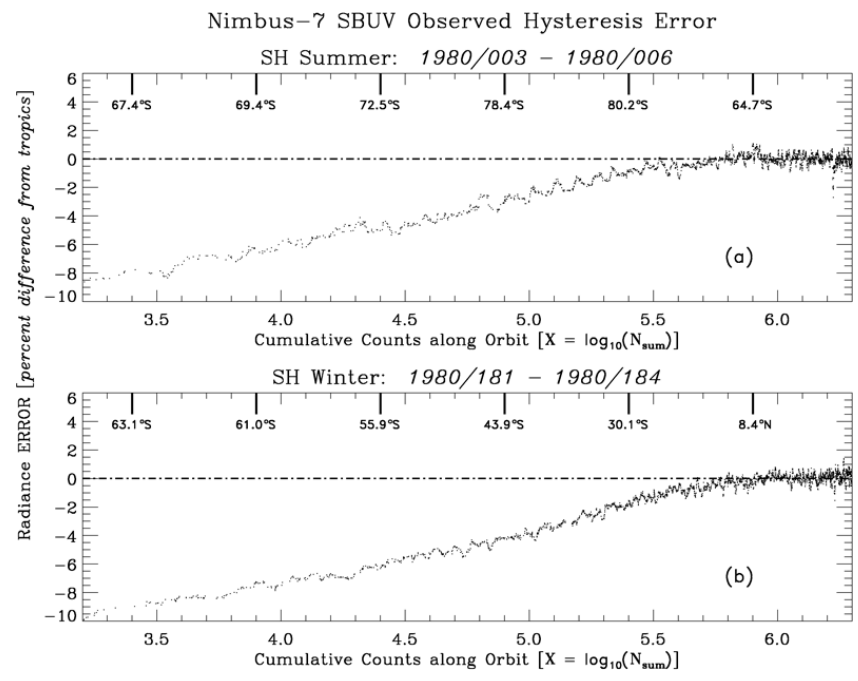

Fig. 13. Hysteresis error for Nimbus-7 SBUV reflectivity measurements, plotted as a function of accumulated counts. The dark tick marks in each panel show the approximate latitude corresponding to the indicated signal level. (a) Average values for 3-6 January 1980. (b) Average values for 29 June-2 July 1980.

\subsection{Out-of-band stray light}

The terrestrial backscattered radiance increases by four orders of magnitude over a narrow wavelength range (250$340 \mathrm{~nm}$ ), as shown in Fig. 5, so that SBUV radiance measurements at short wavelengths are sensitive to out-of-band (OOB) contamination from long wavelength signals. The prelaunch test requirement for SBUV/2 instruments only addressed the magnitude of the signal at $200 \mathrm{~nm}$ with respect to the signal at $400 \mathrm{~nm}$, which all instruments satisfied. Figure 15 shows the spectrum of a ground-based zenith sky measurement with the FM\#8 instrument currently flying on the NOAA-19 spacecraft. The radiance values below $290 \mathrm{~nm}$ should be constant at the instrument noise level due to atmospheric ozone absorption. However, there is a steady increase in the observed signal between $240 \mathrm{~nm}$ and $290 \mathrm{~nm}$, which we attribute to contamination from signals at longer wavelengths. This result is confirmed on-orbit by looking at the correlation between fluctuations in short wavelength albedo, which is expected to change smoothly along the orbit, and concurrent surface reflectivity variations, as shown in Fig. 16. In order to determine the contribution to this contamination signal from nearby wavelengths, we need a model of the SBUV/2 slit function.

Laboratory measurements of mercury lamps with SBUV/2 instruments show "wings" surrounding narrow emission lines such as the strong feature at $253.7 \mathrm{~nm}$. We found that these wings could be characterized with linear segments (on a logarithmic intensity scale) of approximately $70 \mathrm{~nm}$ nominal length added to the base of the nominal slit function (triangular bandpass, $1.1 \mathrm{~nm}$ full width at half-maximum

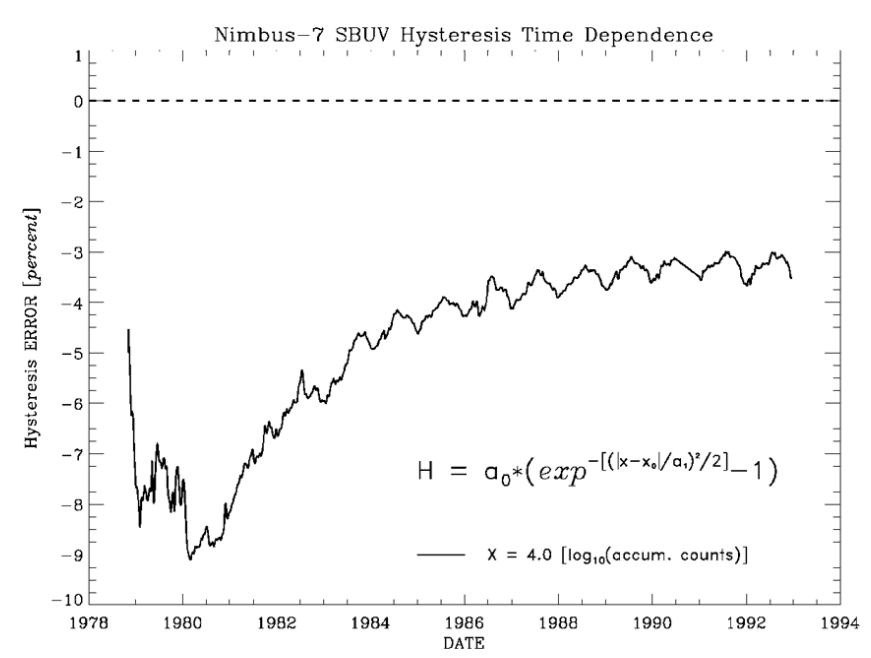

Fig. 14. Time dependence of the Nimbus-7 SBUV hysteresis correction for a signal level of 10000 accumulated counts. The functional form used to parameterize the hysteresis correction is also shown.

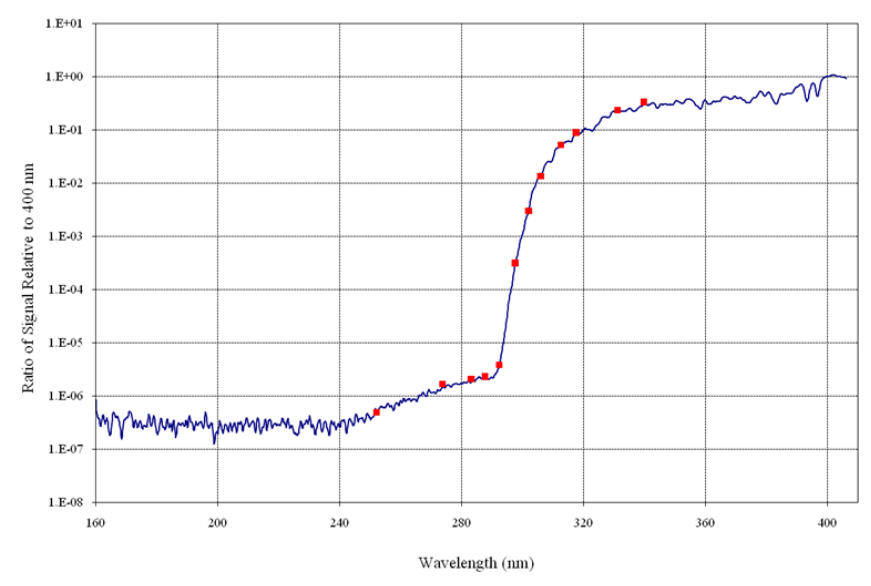

Fig. 15. Zenith sky radiance observed by the SBUV/2 FM\#8 instrument (flown on NOAA-19) during laboratory testing in October 2000 , normalized to the signal at $400 \mathrm{~nm}$. The 12 wavelengths used for normal discrete ozone measurements are indicated by filled squares. Reproduced from Ball Aerospace (2005) with permission of Ball Aerospace and Technology Corporation.

(FWHM)). This form is consistent with the OOB error observed in the prelaunch skylight test.

In order to determine an OOB correction function for each instrument, we generate an appropriate high resolution radiance spectrum using a synthetic albedo spectrum created by the TOMRAD code (Joiner et al., 2004) for specific viewing conditions, a high resolution solar irradiance spectrum measured by UARS SOLSTICE (Rottman et al., 1993), and the spectrally dependent prelaunch radiance sensitivity for that instrument. Least squares fitting of this composite spectrum to the skylight data indicated that slightly longer slit function wings were needed, which was confirmed with onorbit correlation analysis results. The modified slit function 


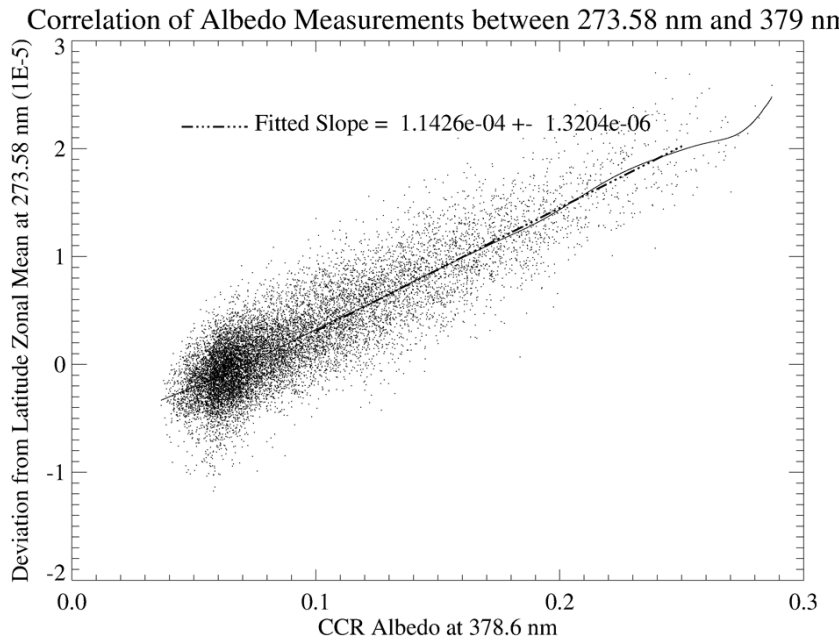

Fig. 16. Scatter plot of NOAA-17 uncorrected albedo deviations from zonal average at $273.5 \mathrm{~nm}$ vs. concurrent CCR measurements. Data are shown for 2 July 2003.

model was then used to calculate OOB errors for different measurement conditions. The magnitude of the OOB error for each scan is parameterized using the observed radiance at $331 \mathrm{~nm}$, since this wavelength falls within the wings of the modified slit function. Observed CCR variations within each scan are used to adjust the calculated OOB error magnitude as necessary for short wavelength channels. The complete OOB error is parameterized as a function of solar zenith angle, reflectivity at $331 \mathrm{~nm}$, nominal pressure level for the reflecting surface, total column ozone, and nominal profile shape (low, middle high latitude) for each scene. Figure 17 shows the spectral dependence of the OOB error for NOAA$17 \mathrm{SBUV} / 2$ for a high contamination scene (SZA $=30^{\circ}$, total ozone $=275 \mathrm{DU}$, reflectivity $=80 \%$ ). Note that the computed OOB error for radiances at operational wavelengths reaches $14 \%$ at $273 \mathrm{~nm}$ for these conditions, then falls well below $1 \%$ for wavelengths longer than $302 \mathrm{~nm}$. The typical uncertainty in this calculated error is approximately $0.2 \%$ or less, as discussed in the Supplement.

\subsection{In-band stray light}

Figure 18 shows the variation of NOAA-17 SBUV/2 $273 \mathrm{~nm}$ albedo values at SZA $>60^{\circ}$ for July 2002. Northern Hemisphere data show a smooth decrease with increasing SZA, falling to zero beyond the terminator $\left(\mathrm{SZA}=90^{\circ}\right)$. However, Southern Hemisphere albedo data increase by $\sim 20$ $25 \%$ over a short range $\left(\mathrm{SZA}=76^{\circ}-78^{\circ}\right)$, and remain elevated on the night side until the satellite passes into Earth shadow at $\mathrm{SZA}=118^{\circ}$. The onset of this behavior is extremely repeatable in solar zenith angle throughout the year, which means that the latitude of onset varies with season. The relative magnitude of the error observed in raw data decreases with increasing wavelength, and is less than $1 \%$

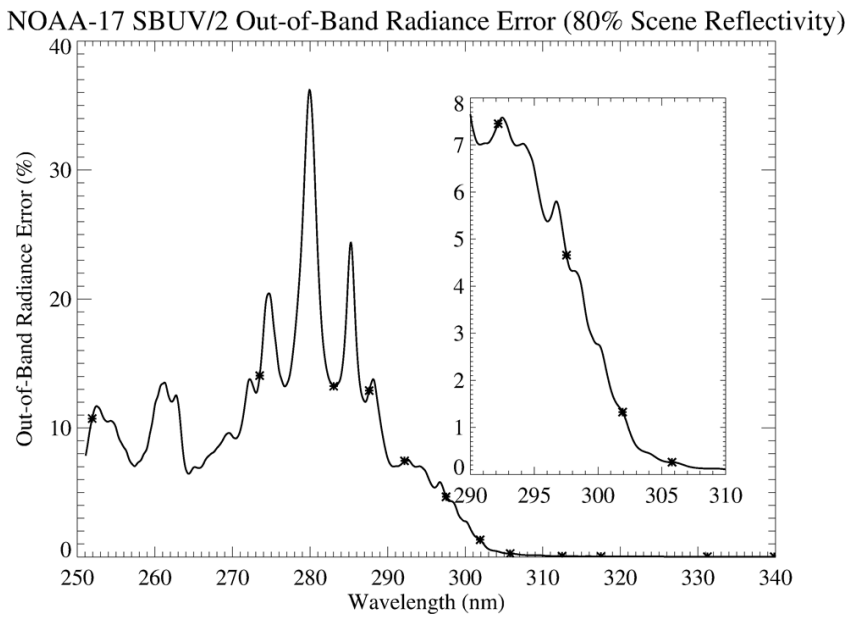

Fig. 17. Calculated out-of-band (OOB) contamination for NOAA$17 \mathrm{SBUV} / 2$ at solar zenith angle $=30^{\circ}$, total ozone $=275 \mathrm{DU}$, reflectivity $=80 \%$. The 12 wavelengths used for normal discrete ozone measurements are indicated by asterisks. The inset panel expands the $290-310 \mathrm{~nm}$ wavelength region.

longward of $300 \mathrm{~nm}$. We use the term "in-band stray light" (IBSL) to distinguish this error from the problem described in Sect. 4.2. The presence of IBSL error at SZA $>90^{\circ}$, when the Earth is in shadow but the Sun still illuminates the spacecraft, demonstrates that the source of the error is the solar signal (as opposed to terrestrial radiance). No corresponding errors are seen in concurrent CCR data, indicating that the problem only affects monochromator measurements.

The NOAA-17 SBUV/2 instrument (FM\#6) used a 4segment depolarizer with glass elements separated by small air gaps, rather than being in optical contact as with earlier SBUV/2 instruments. The same design change was made for the FM\#5, FM\#7, FM\#8 instruments flown on the NOAA-14, NOAA-18, and NOAA-19 spacecraft, respectively, and similar IBSL problems have been observed for each of these instruments. The basic analysis procedure described below for NOAA-17 SBUV/2 has also been applied to determine IBSL corrections for the NOAA-14 and NOAA-18 V8.6 ozone data products. The functional form described here can be extrapolated for use in operational ozone processing, and is updated annually. A more extensive discussion can be found in Huang et al. (2012).

To characterize the IBSL problem, we first look at night side data away from the terminator, where earthshine does not affect the analysis. Measurements at $\mathrm{SZA}=96^{\circ}$ and $\mathrm{SZA}=108^{\circ}$ show an approximately linear variation in magnitude with increasing SZA, as well as a strong seasonal correlation of this slope with spacecraft-centered solar azimuth angle (SCSAA). The IBSL error can be separated into time-dependent and SCSAA-dependent components. Figure 19 shows that the magnitude of the correction function slope (dIBSL/dSZA) night derived from nightside data generally decreases with time. Note that because the relative 


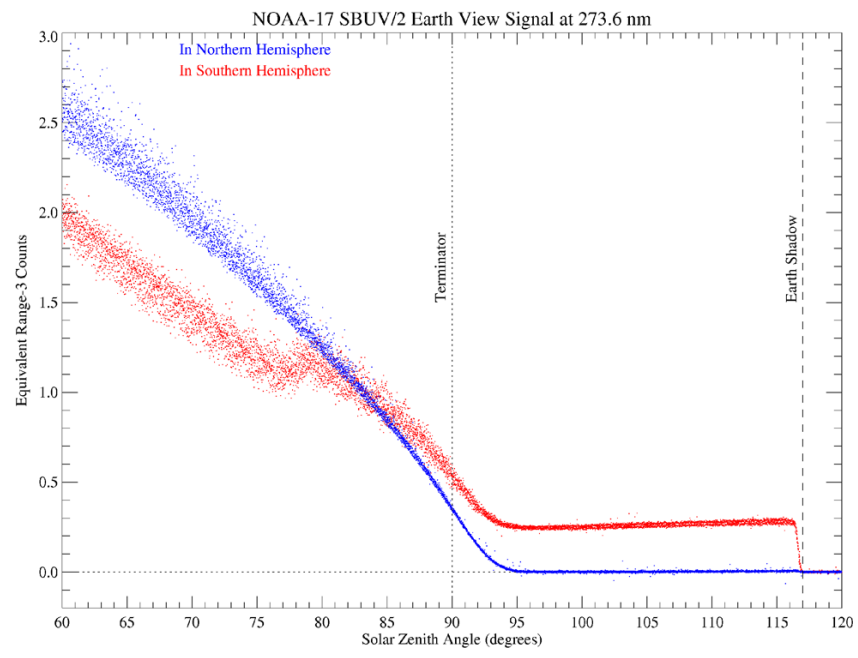

Fig. 18. NOAA-17 SBUV/2 uncorrected radiance data for July 2002 at $273.6 \mathrm{~nm}$ and solar zenith angle $>60^{\circ}$ for Northern Hemisphere measurements (blue) and Southern Hemisphere measurements (red). The onset of the in-band stray light (IBSL) error is evident at $\mathrm{SZA}=76^{\circ}-78^{\circ}$ in the Southern Hemisphere. The satellite passes into the Earth's shadow at $\mathrm{SZA}=117^{\circ}$ while orbiting at an altitude of $810 \mathrm{~km}$.

magnitude of the long wavelength IBSL correction is very small compared to the radiance signal $\left(\sim 10^{-4}\right)$, the positive change of the correction early in the NOAA-17 record for these wavelengths is not significant. The SCSAA dependence can be characterized with a cubic function scaled to each wavelength. As a result, the normalized slope of the correction, (dIBSL/dSZA) night/IBSL, is approximately wavelength-independent.

Extrapolation of the night side IBSL correction functions to the onset of IBSL errors at $\mathrm{SZA}=75^{\circ}$ on the day side gave results that did not agree with the observed radiance errors. We therefore evaluated NOAA-17 day side errors by comparing initial profile residuals with unaffected NOAA16 observations, averaged in $0.5^{\circ}$ latitude bands. These data were used to characterize the background albedo variation prior to the onset of IBSL contamination, and then determine a linear SZA dependence for both the rising section of albedo data $\left(\mathrm{SZA}=76^{\circ}-78^{\circ}\right)$ and the value at $\mathrm{SZA}=80^{\circ}$. We then used a linear fit between the derived IBSL errors at $\mathrm{SZA}=80^{\circ}$ and $\mathrm{SZA}=96^{\circ}$ to determine the correction function slope (dIBSL/dSZA) day, which is used to calculate the error throughout this SZA range. Since measurement noise limits this analysis to shorter wavelengths, we derive the average of (dIBSL/dSZA) day for the four shortest wavelengths and assume that the normalized correction slope is still wavelength-independent. We force the day side function to merge with the night side results at $\mathrm{SZA}=96^{\circ}$ to calculate an IBSL correction for all wavelengths.

We estimate that the uncertainty in the IBSL correction function for each affected instrument is less than $10 \%$ of

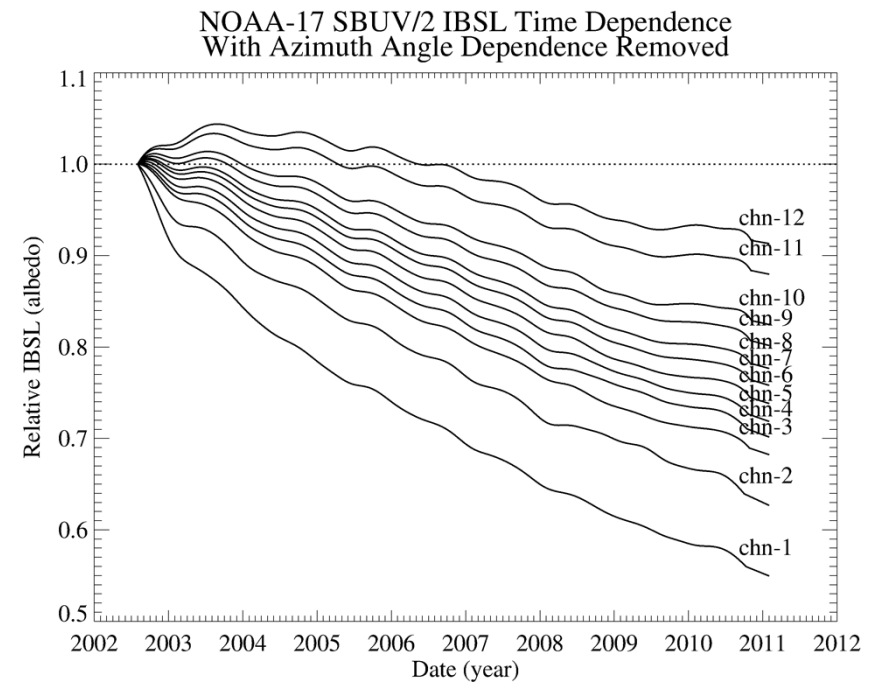

Fig. 19. Time dependence of linear slope (dIBSL/dSZA) night characterizing solar zenith angle dependence of NOAA-17 IBSL error on the night side. All values have been normalized to the initial results derived in August 2002. The seasonal variation in slope due to orbital change has been removed.

the overall correction, so that the uncertainty due to this correction for short wavelength radiance data (most important for calculating upper profile ozone) is approximately 1 $2 \%$. This additional uncertainty is only present for a limited subset of the NOAA-17 measurements where SZA $>75^{\circ}$ is experienced, occurring above $60^{\circ}-65^{\circ} \mathrm{S}$ latitude in summer months and extending down to $\sim 45^{\circ} \mathrm{S}$ latitude in winter months. Note that while NOAA-17 IBSL corrections are applied in the Southern Hemisphere, NOAA-18 IBSL corrections are applied in the Northern Hemisphere. NOAA14 IBSL effects were observed in the Northern Hemisphere while the satellite was in an afternoon Equator-crossing orbit, then shifted to the Southern Hemisphere when it drifted past the terminator.

\subsection{SBUV/2 grating drive errors}

The SBUV/2 instruments use a grating drive with an optical encoder to select each wavelength sequentially during an individual scan. The position reached by the grating drive for each sample is recorded. Due to mechanical problems, some instruments have experienced wavelength selection problems where the indicated measurement location differs from the intended value by one or more grating positions $(1 \mathrm{GPOS}=0.075 \mathrm{~nm})$. The NOAA- 9 and NOAA$11 \mathrm{SBUV} / 2$ instruments had relatively minor grating drive problems that developed later in each instrument's lifetime, with typical errors of $\pm 1-2$ GPOS. The NOAA-14 SBUV/2 instrument had more severe grating drive problems (multiple channels affected, frequent errors of $\triangle \mathrm{GPOS}= \pm 3-4$ ) that began to appear a few months after launch. These errors 
halted operations on a few occasions in 1996 and 1998, and eventually forced the channel 5 wavelength to be changed from $292.3 \mathrm{~nm}$ to $295.0 \mathrm{~nm}$ in June 1998. The SBUV/2 grating drive was modified after the launch of NOAA-14 to improve its long-term stability. Among more recent instruments, only NOAA-17 has developed \pm 1 GPOS errors at channel $12(339.8 \mathrm{~nm})$, and these errors have minimal impact on derived ozone values because that channel is not used in the ozone retrieval.

Grating drive errors are characterized for V8.6 ozone processing by calculating daily average errors based on all measurements at each wavelength. The albedo values in each scan are then corrected for wavelength selection error using interpolation of the nominal ozone cross-section and solar irradiance spectra. Note that the actual wavelength for an individual sample may still be in error by up to \pm 0.5 GPOS $(0.038 \mathrm{~nm})$ because of digitization in the grating drive readout, which could lead to increased errors in the retrieved ozone profile. The overall impact in the data set of any uncorrected grating drive errors can depend on the magnitude of the error, the frequency of occurrence, and the number and spectral location of the channels affected. The impact of such uncorrected errors in the ozone product is typically reduced with spatial and/or temporal averaging.

\subsection{Nimbus-7 chopper non-sync}

The Nimbus-7 SBUV chopper wheel began to lose synchronization with the counting electronics on 13 February 1987. Measurements made after this date were characterized by significantly higher noise in each scan. A correction function was developed based on the coherence of coincident $343 \mathrm{~nm}$ photometer data, as described by Gleason and McPeters (1995). This correction decreased monthly average Umkehr layer ozone standard deviation values by $20-50 \%$. Nimbus-7 profile ozone data during the "non-sync" period (1987-1990) do not show a bias relative to the 1978-1986 data. However, anomalous profiles are much more likely to occur during this period, and users should be cautious when examining individual ozone profiles.

\section{Overall data set uncertainty and quality}

The individual SBUV V8.6 data sets described in this paper can be used to create a continuous 32-yr ozone data record from 1978 through 2010, following the calibration chain shown previously in Fig. 11. We can define two groups of data sets, one with calibrations referenced back to NOAA-11, and the other with calibrations referenced back to NOAA-17. In order to estimate an overall long-term uncertainty for the V8.6 record, we first examined the overlap period between NOAA-11 and NOAA-14 during 1998-2000, where different calibration techniques were used for each instrument. Applying the NLTD approach discussed in Sect. 3.4 to these mea-
Table 1. Long-term albedo uncertainty at selected wavelengths for hypothetical merged V8.6 ozone data set.

\begin{tabular}{llll}
\hline $\begin{array}{l}\text { Data } \\
\text { Set }\end{array}$ & $\begin{array}{l}\text { Time } \\
\text { Period }\end{array}$ & $\begin{array}{l}\text { Channel 2 } \\
\text { Uncertainty }\end{array}$ & $\begin{array}{l}\text { Channel 11 } \\
\text { Uncertainty }\end{array}$ \\
\hline Nimbus-7 & $1978-1989$ & $1.6 \%$ & $1.1 \%$ \\
NOAA-11 & $1989-1995$ & $0.6 \%$ & $0.6 \%$ \\
NOAA-14 & $1995-2005$ & $1.1 \%$ & $1.1 \%$ \\
NOAA-17 & $2005-2010$ & $0.8 \%$ & $0.8 \%$ \\
overlap & $1998-2000$ & $0.6 \%$ & $1.2 \%$ \\
RSS Sum & $1978-2010$ & $2.3 \%$ & $2.2 \%$ \\
\hline
\end{tabular}

surements produces an average albedo calibration difference of $1.2( \pm 1.0) \%$ in this overlap period, with smaller values at shorter wavelengths.

Determining an overall V8.6 data set uncertainty depends in part on how the combined data set is constructed. Table 1 presents an arbitrary choice of data sets that spans the complete data record, along with the estimated time-dependent albedo uncertainty values for chan. $2(273.5 \mathrm{~nm})$ and chan. 11 $(331.2 \mathrm{~nm})$ as derived in the Supplement. The NOAA-11 uncertainty values are reduced from those listed in the Supplement because only part of that data record would be used. Including the overlap uncertainty values as derived above, we calculate an estimated albedo uncertainty of $2.3 \%$ for chan. 2 and $2.2 \%$ for chan. 11 over the 32 -yr data record. This result does not include the absolute uncertainty for any instrument.

An alternate approach to calculating the overall uncertainty is to begin with the absolute uncertainty values for the NOAA-17 and SSBUV instruments. We extend the NOAA17 uncertainty forward to the beginning of the NOAA-14 record using time-dependent and NLTD values for NOAA16 and NOAA-14. We extend the SSBUV uncertainty forward to the beginning of the Nimbus-7 record using the timedependent and coincidence uncertainty values for NOAA11 and Nimbus-7. Combining these quantities gives an estimated albedo uncertainty of $2.8 \%$ for chan. 2 and $2.9 \%$ for chan. 11 over the complete data record. The uncertainty values derived using these different methods should be viewed as an approximate guide, rather than an exact result to be used for any merged V8.6 data set.

There is no single determining factor that defines the "best" choice of SBUV V8.6 data sets to use when more than one instrument is available. In order to provide some quantitative recommendation as to how to best use V8.6 data in such situations, we define two parameters from the final residual values to help indicate the relative quality of the retrieved ozone profile. Res_Long represents the range (maximum - minimum) of the final residuals for the three longest wavelengths used in a retrieval, and provides information about the lower portion of the ozone profile. Res_Short represents the range of the final residuals for all shorter wavelengths used in a retrieval, and provides information about 


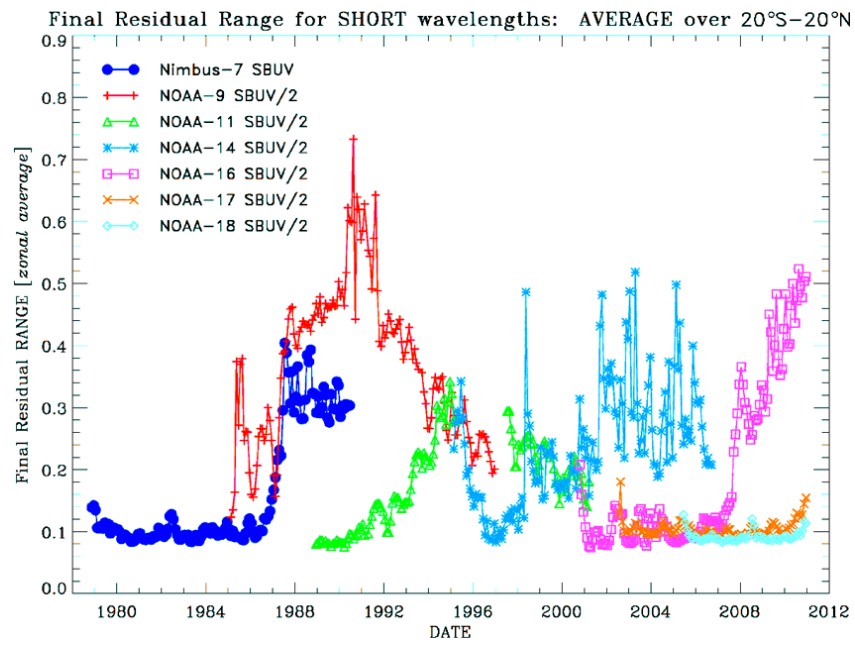

Fig. 20. Time series of final residual range for the shortest wavelengths used in the V8.6 profile ozone retrieval (Res_Short). All values are averaged over each month and $20^{\circ} \mathrm{S}-20^{\circ} \mathrm{N}$ latitude.

the upper portion of the ozone profile. We have calculated monthly average values of Res_Long and Res_Short averaged between $20^{\circ} \mathrm{S}$ and $20^{\circ} \mathrm{N}$ in order to provide a relative assessment of data quality between different instruments. Figure 20 shows the averaged Res_Short time series values for all overlapping instruments, and Fig. 21 shows the corresponding averaged Res_Long time series values. A moderate increase in averaged residual range values is typically observed at $\mathrm{SZA}>70^{\circ}$, which corresponds to terminator-crossing periods for the low latitudes used to create these figures. Specific features visible for individual instruments include the onset of Nimbus-7 chopper non-sync problems in 1987, volcanic eruption effects at long wavelengths in 1982 (El Chichón) and 1991 (Mt. Pinatubo), and NOAA-14 scene brightness change representation in 1995. NOAA-9 residual range values are frequently higher than overlapping instruments, and are not recommended as a primary data choice when other measurements are available.

\section{Conclusions}

The SBUV V8.6 data set represents ozone data products from 8 different instruments covering more than $40 \mathrm{yr}$. We have analyzed prelaunch measurements, on-orbit calibration system data, and carefully selected operational measurements to produce radiance-based absolute and time-dependent characterizations of these instruments. We supplement the laboratory and on-orbit calibration measurements with "soft" calibration techniques that improve the accuracy of the retrieved ozone profiles. In particular, we have used soft calibration to determine small absolute adjustments to individual channels for NOAA-17 that let us use those data as a baseline for intercalibration of other instruments. Analysis of measure-

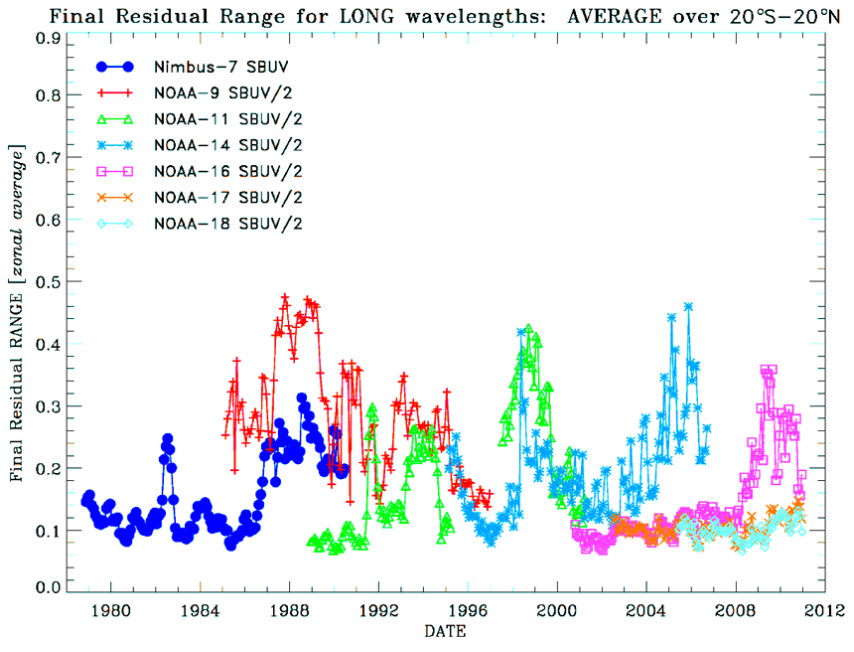

Fig. 21. Time series of final residual range for the three longest wavelengths used in the V8.6 profile ozone retrieval (Res_Long). All values are averaged over each month and $20^{\circ} \mathrm{S}-20^{\circ} \mathrm{N}$ latitude.

ments of Antarctic snow and ice-covered regions at $340 \mathrm{~nm}$ provides instrument sensitivity change information that complements the solar measurements and on-board diffuser calibration system, and in some cases yields a better long-term characterization.

The temporal overlap between 7 of these data sets allows us to further inter-calibrate these instruments to create a continuous 32-yr ozone data record for long-term trend studies. The more recent SBUV/2 instruments (NOAA-16, NOAA17, NOAA-18) have better data quality than earlier SBUV/2 instruments due to improvements in instrument design and less orbit drift. We focused on concurrent measurements with no local time difference between two instruments to develop calibration adjustments that link these overlapping data sets together. We estimate the long-term uncertainty of the albedo values used in the V8.6 retrieval algorithm for a single wavelength to be approximately $3 \%$ or less over the length of this combined data record.

\section{Supplementary material related to this article is available online at: http://www.atmos-meas-tech.net/5/ 2951/2012/amt-5-2951-2012-supplement.pdf.}

Acknowledgements. We gratefully acknowledge the efforts of many people who have contributed to the calibration of the SBUV instruments over the past 40 years. This work was supported by NASA through contract NNH06HX18C.

Edited by: M. Weber 


\section{References}

Ball Aerospace Engineering Division: Specification Compliance and Calibration Data Book for SBUV/2 Flight Model \#6, Mod. 8, IN021-A-035, Rev. A, October 2005.

Bhartia, P. K., McPeters, R. D., Flynn, L. E., Taylor, S., Kramrova, N. A., Frith, S., Fisher, B., and DeLand, M.: Solar Backscatter UV (SBUV) total ozone and profile algorithm, Atmos. Meas. Tech. Discuss., 5, 5913-5951, doi:10.5194/amtd-5-5913-2012, 2012.

Boyd, I. S., Parrish, A. D., Froidevaux, L., von Clarmann, T., Kyrölä, E., Russell III, J. M., and Zawodny, J. M.: Groundbased microwave ozone radiometer measurements, compared with Aura-MLS v2.2 and other instruments at two Network for Detection of Atmospheric Composition Change sites, J. Geophys. Res., 112, D24S33, doi:10.1029/2007JD008720, 2007.

Butler, J. J., Park, H., Barnes, P. Y., Early, E. A., van Eijk-Olij, C., Zantman, A. E., van Buller-Leeuwen, S., and Schaarsberg, J. G.: Comparison of ultraviolet bidirectional reflectance distribution function (BRDF) measurements of diffusers used in the calibration of the Total Ozone Mapping Spectrometer (TOMS), Proc. SPIE Int. Soc. Opt. Eng., 4881, 345-354, 2003.

Cao, C., Weinreb, M., and Xu, H.: Predicting simultaneous nadir overpasses among polar-orbiting satellites for the intersatellite calibration of radiometers, J. Atmos. Ocean. Tech., 21, 537-542, 2004.

Cebula, R. P., Park, H., and Heath, D. F.: Characterization of the Nimbus-7 SBUV radiometer for the long-term monitoring of stratospheric ozone, J. Atmos. Oceanic Technol., 5, 215-227, 1988.

Cebula, R. P., DeLand, M. T., Hilsenrath, E., Schlesinger, B. M., Hudson, R. D., and Heath, D. F.: Intercomparisons of the solar irradiance measurements from the Nimbus-7 SBUV, the NOAA-9 and NOAA-11 SBUV/2, and the STS-34 SSBUV instruments: A preliminary assessment, J. Atmo. Terr. Phys., 53, 993-997, 1991.

Cebula, R. P., Thullier, G. O., VanHoosier, M. E., Hilsenrath, E., Herse, M., Brueckner, G. E., and Simon, P. C.: Observations of the solar irradiance in the $200-350 \mathrm{~nm}$ interval during the ATLAS-1 mission: A comparison among three sets of measurements-SSBUV, SOLSPEC, and SUSIM, Geophys. Res. Lett., 23, 2289-2292, 1996.

DeLand, M. T. and Cebula, R. P.: Composite Mg II solar activity index for solar cycles 21 and 22, J. Geophys. Res., 98, 1280912823, 1993.

DeLand, M. T., Cebula, R. P., Huang, L.-K., Taylor, S. L., Stolarski, R. S., and McPeters, R. D.: Observations of "hysteresis" in backscattered ultraviolet ozone data, J. Atmos. Oceanic Technol., 18, 914-924, 2001.

Fioletov, V. E., Tarasick, D. W., and Petrapavlovskikh, I.: Estimating ozone variability and instrument uncertainties from SBUV(/2), ozonesonde, Umkehr, and SAGE II measurements: Short-term variations, J. Geophys. Res., 111, D02305, doi:10.1029/2005JD006340, 2006.

Frederick, J. E., Cebula, R. P., and Heath, D. F.: Instrument characterization for the detection of long-term changes in stratospheric ozone: An analysis of the SBUV/2 radiometer, J. Atmos. Oceanic Technol., 3, 472-480, 1986.

Frederick, J. E., Niu, X., and Hilsenrath, E.: An approach to the detection of long-term trends in upper stratospheric ozone from space, J. Atmos. Oceanic Technol., 7, 734-740, 1990.
Gleason, J. F., and McPeters, R. D.: Corrections to the Nimbus 7 solar backscatter ultraviolet data in the "nonsync" period (February 1987 to June 1990), J. Geophys. Res., 100, 16873-16877, 1995.

Haefele, A., Hocke, K., Kämpfer, N., Keckhut, P., Marchand, M., Bekki, S., Morel, B., Egorova, T., and Rozanov, E.: Diurnal changes in middle atmospheric $\mathrm{H}_{2} \mathrm{O}$ and $\mathrm{O}_{3}$ : Observations in the Alpine region and climate models, J. Geophys. Res., 113, D17303, doi:10.1029/2008JD009892, 2008.

Heath, D. F. and Healy, J. B.: Observations on degradation of ultraviolet systems on Nimbus spacecraft, in Space Optics, Nat. Acad. Sci., Washington, DC, 340-354, 1974.

Heath, D. F., Krueger, A. J., Roeder, H. A., and Henderson, B. D.: The Solar Backscatter Ultraviolet and Total Ozone Mapping Spectrometer (SBUV/TOMS) for Nimbus G, Opt. Eng., 14, 323331, 1975.

Heath, D. F., Wei, Z., Fowler, W. K., and Nelson, V. W.: Comparison of spectral radiance calibrations of SBUV-2 satellite ozone monitoring instruments using integration sphere and flat-plate diffuser techniques, Metrologia, 30, 259-264, 1993.

Herman, J. R., Hudson, R. D., and Serafino, G.: Analysis of the eight-year trend in ozone depletion from empirical models of Solar Backscattered Ultraviolet instrument degradation, J. Geophys. Res., 95, 7403-7416, 1990.

Herman, J. R., Hudson, R., McPeters, R., Stolarski, R., Ahmad, Z., $\mathrm{Gu}, \mathrm{X} .-$ Y., Taylor, S., and Wellemeyer, C.: A new self-calibration method applied to TOMS and SBUV backscattered ultraviolet data to determine long-term global ozone change, J. Geophys. Res., 96, 7531-7545, 1991.

Hilsenrath, E., Cebula, R. P., DeLand, M. T., Laamann, K., Taylor, S., Wellemeyer, C., and Bhartia, P. K.: Calibration of the NOAA 11 SBUV/2 ozone data set from 1989 to 1993 using in-flight calibration data and SSBUV, J. Geophys. Res., 100, 1351-1366, 1995.

Huang, L.-K., Cebula, R. P., Taylor, S. L., DeLand, M. T., McPeters, R. D., and Stolarski, R. S.: Determination of NOAA-11 SBUV/2 radiance sensitivity drift based on measurements of polar ice cap radiance, Int. J. Remote. Sens., 24, 305-314, 2003.

Huang, L. K., Flynn, L. E., and DeLand, M. T.: Derivation of inband stray light correction for NOAA-17 SBUV/2, Atmos. Meas. Tech., in preparation, 2012.

Janz, S., Hilsenrath, E., Butler, J., Heath, D. F., and Cebula, R. P.: Uncertainties in radiance calibrations of backscatter ultraviolet (BUV) instruments, Metrologia, 32, 637-641, 1995.

Jaross, G. and Krueger, A. J.: Ice radiance method for BUV instrument monitoring, Proc. SPIE Int. Soc. Opt. Eng., 2047, 94-101, 1993.

Jaross, G. and Warner, J.: Use of Antarctica for validating reflected solar radiation measured by satellite sensors, J. Geophys. Res., 113, D16S34, doi:10/1029/2007JD008835, 2008.

Joiner, J., Vasilkov, A. P., Flittner, D. E., Gleason, J. F., and Bhartia, P. K.: Retrieval of cloud pressure and oceanic chlorophyll content using Raman scattering in GOME ultraviolet spectra, J. Geophys. Res., 109, D01109, doi:10.1029/2003JD003698, 2004.

McPeters, R. D.: The behavior of ozone near the stratopause from two years of BUV observations, J. Geophys. Res., 85, 45454550, 1980.

McPeters, R. D. and Labow, G. J.: Climatology 2011: An MLS and sonde derived ozone climatology for satellite retrieval algorithms, J. Geophys. Res., 117, D10303, 
doi:10.1029/2011JD017006, 2012.

McPeters, R., Bhartia, P. K., Haffner, D., and Labow, G.: A long term SBUV ozone data record: 1970-2011, J. Geophys. Res., in preparation, 2012.

Price, J. C.: Timing of NOAA afternoon passes, Int. J. Rem. Sens., 12, 193-198, 1991.

Rottman, G. J., Woods, T. N., and Sparn, T. P.: Solar-stellar irradiance comparison experiment 1: 1. Instrument design and operation, J. Geophys. Res., 98, 10667-10677, 1993.

Schlesinger, B. M. and Cebula, R. P.: Solar variation 1979-1987 estimated from an empirical model for changes with time in the sensitivity of the Solar Backscatter Ultraviolet instrument, J. Geophys. Res., 97, 10119-10134, 1992.

Stajner, I., Winslow, N., Rood, R. B., and Pawson, S.: Monitoring of observation errors in the assimilation of satellite ozone data, J. Geophys. Res., 109, D06309, doi:10.1029/2003JD004118, 2004.

Stewart, T. B., Arnold, G. S., Hall, D. F., and Marten, H. D.: Absolute rates of vacuum-ultraviolet photochemical deposition of organic films, J. Phys. Chem., 93, 2393-2407, 1989.
Stolarski, R. S. and Frith, S. M.: Search for evidence of trend slowdown in the long-term TOMS/SBUV total ozone data record: the importance of instrument drift uncertainty, Atmos. Chem. Phys., 6, 4057-4065, doi:10.5194/acp-6-4057-2006, 2006.

Stolarski, R. S., Labow, G. J., and McPeters, R. D.: Springtime Antarctic total ozone measurements in the early 1970s from the BUV instrument on Nimbus 4, Geophys. Res. Lett., 24, 591-594, 1997.

Terao, Y. and Logan, J. A.: Consistency of time series and trends of stratospheric ozone as seen by ozonesonde, SAGE II, HALOE, and SBUV(/2), J. Geophys. Res., 112, D06310, doi:10.1029/2006JD007667, 2007.

Walker, J. H., Saunders, R. D., Jackson, J. K., and McSparron, D. A.: Spectral Irradiance Calibrations, NBS Special Publication 250-20, National Bureau of Standards, Gaithersburg, MD, 102 pp., 1987.

Weiss, H., Cebula, R. P., Laamann, K., and Hudson, R. D.: Evaluation of the NOAA 11 solar backscatter ultraviolet radiometer, mod 2 (SBUV/2): Inflight calibration, Proc. SPIE Int. Soc. Opt. Eng., 1493, 80-90, 1991. 